\title{
20E-mediated regulation of BmKr-h1 by BmKRP promotes oocyte maturation
}

\author{
Zidan Zhu', Chunmei Tong ${ }^{1}$, Binbin Qiu', Hongguang Yang ${ }^{1}$, Jiahui Xu', Sichun Zheng ${ }^{1}$, Qisheng Song ${ }^{2}$, \\ Qili Feng ${ }^{1 *}$ and Huimin Deng ${ }^{1 *}$ (D)
}

\begin{abstract}
Background: Krüppel homolog $1(\mathrm{Kr}-\mathrm{h} 1)$ is a critical transcription factor for juvenile hormone $(\mathrm{JH})$ signaling, known to play a key role in regulating metamorphosis and adult reproduction in insects. Kr-h1 can also be induced by molting hormone 20-hydroxyecdysone (20E), however, the underlying mechanism of 20E-induced Kr-h1 expression remains unclear. In the present study, we investigated the molecular mechanism of $\mathrm{Kr}-\mathrm{h} 1$ induction by $20 \mathrm{E}$ in the reproductive system of a model lepidopteran insect, Bombyx mori.

Results: Developmental and tissue-specific expression analysis revealed that BmKr-h1 was highly expressed in ovaries during the late pupal and adult stages and the expression was induced by 20E. RNA interference (RNAi)mediated depletion of BmKr-h1 in female pupae severely repressed the transcription of vitellogenin receptor (VgR), resulting in the reduction in vitellogenin $(\mathrm{Vg})$ deposition in oocytes. BmKr-h1 specifically bound the Kr-h1 binding site (KBS) between - 2818 and $-2805 \mathrm{nt}$ upstream of $B m V g R$ and enhanced the transcription of BmVgR. A $20 \mathrm{E}$ cisregulatory element (CRE) was identified in the promoter of $B m K r-h 1$ and functionally verified using luciferase reporter assay, EMSA and DNA-ChIP. Using pull-down assays, we identified a novel transcription factor B. mori Kr-h1 regulatory protein (BmKRP) that specifically bound the BmKr-h1 CRE and activated its transcription. CRISPR/Cas9mediated knockout of BmKRP in female pupae suppressed the transcription of $B m K r-h 1$ and $B m V g R$, resulting in arrested oogenesis.
\end{abstract}

Conclusion: We identified BmKRP as a new transcription factor mediating 20E regulation of B. mori oogenesis. Our data suggests that induction of BmKRP by 20 E regulates BmKr-h1 expression, which in turn induces BmVgR expression to facilitate $\mathrm{Vg}$ uptake and oogenesis.

Keywords: 20-hydroxyecdysone, Krüppel homolog 1, Kr-h1 regulatory protein, Vitellogenin receptor, Oogenesis

\section{Background}

Juvenile hormone $(\mathrm{JH})$ and 20-hydroxyecdysone (20E) regulate many physiological processes, including the development, metamorphosis and reproduction of insects [1]. During larval stages, JH binds its receptor Methoprene-

\footnotetext{
*Correspondence: qlfeng@scnu.edu.cn; denghuiminmin@163.com ${ }^{1}$ Guangdong Key Laboratory of Insect Developmental Biology and Applied Technology, Guangzhou Key Laboratory of Insect Development Regulation and Application Research, Institute of Insect Science and Technology \& School of Life Sciences, South China Normal University, Guangzhou 510631, China

Full list of author information is available at the end of the article
}

tolerant (MET) to initiate the $\mathrm{JH}$ signaling pathway, suppressing metamorphosis until larvae reach the appropriate size and development stage. In the final instar, JH titers decrease while 20E levels increase to induce larval-pupal metamorphosis $[1-3]$. JH and $20 \mathrm{E}$ also regulate many aspects of reproduction, including previtellogenic development, vitellogenesis and oogenesis [4-7]. Vitellogenesis is a critical event of female reproduction, in which vitellogenin $(\mathrm{Vg})$ is synthesized in fat body, secreted into hemolymph and then taken up by developing oocytes via receptor-mediated endocytosis $[8,9]$.

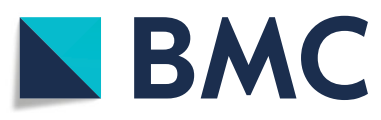

(c) The Author(s). 2021 Open Access This article is licensed under a Creative Commons Attribution 4.0 International License, which permits use, sharing, adaptation, distribution and reproduction in any medium or format, as long as you give appropriate credit to the original author(s) and the source, provide a link to the Creative Commons licence, and indicate if changes were made. The images or other third party material in this article are included in the article's Creative Commons licence, unless indicated otherwise in a credit line to the material. If material is not included in the article's Creative Commons licence and your intended use is not permitted by statutory regulation or exceeds the permitted use, you will need to obtain permission directly from the copyright holder. To view a copy of this licence, visit http://creativecommons.org/licenses/by/4.0/. The Creative Commons Public Domain Dedication waiver (http://creativecommons.org/publicdomain/zero/1.0/) applies to the data made available in this article, unless otherwise stated in a credit line to the data. 
Krüppel homolog 1 (Kr-h1), a transcription factor containing a DNA binding domain with eight cystine 2 histidine $2\left(\mathrm{C}_{2} \mathrm{H}_{2}\right)$-type zinc $(\mathrm{Zn})$-fingers, is induced by $\mathrm{JH}$ and functions in the $\mathrm{JH}$ signal transduction pathway. The properties of $K r-h 1$ as a transducer of the anti-metamorphic action of $\mathrm{JH}$ are conserved in hemimetabolous and holometabolous insects [10-15]. $K r-h 1$ can also be induced by $20 \mathrm{E}$, and in fact, $K r-h 1$ was first identified as a stage-specific modulator of the prepupal ecdysone response at the onset of Drosophila melanogaster metamorphosis [16]. DmKr-h1 was upregulated 2 -fold at $6 \mathrm{~h}$ post $20 \mathrm{E}$ treatment and 3.5 -fold at $6 \mathrm{~h}$ post $20 \mathrm{E}$ and cycloheximide treatment in the cultured partial blue gut of 3rd instar larvae [17]. Exposure of Tribolium castaneum cells to $10 \mu \mathrm{M}$ $20 \mathrm{E}$ induced the expression of $K r-h 1$ [18]. In Helicoverpa armigera, $\mathrm{HaKr}$-h1 was upregulated 2.0-fold at $24 \mathrm{~h}$ post $20 \mathrm{E}$ treatment in the 4 th instar larval epidermis [19]. Treatment of 20E in the early 4th larval instar of the recessive trimolter European No. $7 \mathrm{mu}-$ tant of Bombyx mori maintains continuous expression of $B m K r-h 1, \quad$ suppresses premature induction of broad-complex (BR-C Z1), and then induced an additional larval instar [20]. In B. mori, 20E synergized $B m K r-h 1$ induction by $\mathrm{JH}$ analog (JHA) methoprene in the cultured epidermis of 4th instar larvae and pupae [3, 15]. Based on the above data, the induced expression of $K r-h 1$ by $20 \mathrm{E}$ exists in a variety of insects. However, the molecular mechanisms and physiological functions of 20E-mediated $B m K r-h 1$ induction remain unknown.

In addition to repressing metamorphosis, $K r-h 1$ has also been found to function in female reproduction. In Locusta migratoria, Nilaparvata lugens, Aedes aegypti, $H$. armigera, Bactrocera dorsalis, and Sogatella furcifera, silencing $K r$ - $h 1$ blocks JH-regulated expression of $\mathrm{Vg}$, oocyte maturation and ovarian development, and reduces egg production [19, 21-25]. However, in Pyrrhocoris apterus and $T$. castaneum, although depletion of Met suppresses the $V g$ expression in fat body, silencing $K r-h 1$ has no effect on $V g$ expression $[14,26]$. In Cimex lectularius, knocking down of $K r-h 1$ in adult females does not reduce the number of eggs but severely affects egg hatching [27]. Thus, the function of $K r-h 1$ in female reproduction varies widely among insect species, and the molecular mechanism of $K r-h 1$ in reproduction remains largely unknown.

In the present study, a $20 \mathrm{E}$ cis-regulatory element (CRE) in the promoter region of $B m K r-h 1$ and a novel $K r-h 1$ regulatory protein (BmKRP) that binds this CRE were identified. Furthermore, in responding to $20 \mathrm{E}$ stimulation, BmKRP was shown to induce $B m K r-h 1$ expression, which in turn regulated ovarian development and oogenesis via $\mathrm{Vg}$ receptor (BmVgR). Our data demonstrated that 20E-induced BmKRP mediated $\mathrm{Kr}-\mathrm{hl}$ expression in the female reproduction of B. mori.

\section{Results \\ Developmental and 20E-induced expression of $B m K r-h 1$ in ovaries}

The developmental and 20E-induced expression profiles of $B m K r-h 1$ in the ovaries were determined by quantitative real-time PCR (qRT-PCR). Two isoforms of $B m K r$ $h 1, B m K r-h 1 \alpha$ (AB360766) and BmKr-h1 $\beta$ (AB642242), have been reported [28]. Compared to $B m K r-h 1 \beta$, $B m K r-h 1 \alpha$ is predominant in the larval epidermis and is induced by JHA in the epidermis of day 0 of 4 th instar allatectomized larvae and in NIAS-Bm-aff3 cells, but $B m K r-h 1 \beta$ is not sensitive to JHA induction [28]. The expression of $B m K r-h 1 \alpha$ was also detected predominantly in the ovaries comparing to $B m K r-h 1 \beta$ (Additional file 1, Fig. S1A and B) and its mRNA levels were low in the prepupal stage, gradually increased until day 7 of the pupal stage, then dramatically increased on day 8 of pupal stage and remained high during the adult stage (Fig. 1a), which are in general correlated with the peaks of $20 \mathrm{E}$ titers as reported previously $[29,30]$. Since JH is absent during the late pupal stage, these results suggest that $B m K r-h 1 \alpha$ might be induced by $20 \mathrm{E}$ through an unknown transcription factor. To test this hypothesis, the effect of $20 \mathrm{E}$ on the expression of $B m K r-h 1$ was examined in the cultured ovaries isolated from 5-day-old pupae and $\mathrm{BmN}$ cells. The result showed clearly that the expression of $B m K r-h 1 \alpha$ was induced by $20 \mathrm{E}$ at a physiological concentration of $1 \mu \mathrm{M}$ in the cultured ovaries and reached the peak at $4 \mathrm{~h}$ post $20 \mathrm{E}$ treatment (Fig. $1 \mathrm{~b}, \mathrm{c}$ ). In $\mathrm{BmN}$ cells, 20E induced $B m K r-h 1 \alpha$ in a time- and dose-dependent manner (Fig. 1d, e). Although the $B m K r-h 1 \beta$ expression was also induced by $20 \mathrm{E}$ treatment in the cultured ovaries and BmN cells, the mRNA levels were much lower than those of $B m K r-h 1 \alpha$ (Additional file 1, Fig. S1B and C). Therefore, $B m K r-h 1 \alpha$ was used for in-depth studies in the subsequent experiments.

\section{$B m K r-h 1$ upregulated the $B m V g R$ transcription in the oocyte development}

To investigate the function of $B m K r-h 1$ induced by $20 \mathrm{E}$ in the female reproduction, $B m K r-h 1$ dsRNA was injected into the wild-type pupae on day 6. qRT-PCR analysis showed that the $B m K r-h 1$ dsRNA efficiently reduced $B m K r-h 1$ transcript abundance in the ovaries 24 and $48 \mathrm{~h}$ post dsRNA treatment (Fig. 2A). Totally, $13 \%$ of the oocytes in the ovaries of ds $B m K r$ - $h 1$-treated 8day-old pupae had less yolk protein deposition and partially transparent chorion, whereas there were none of these abnormal oocytes in the ovaries of the control (dsEGFP treated) (Fig. 2B and Additional file 1, Table $\mathrm{S} 1$ ). These abnormal eggs gradually became black and 

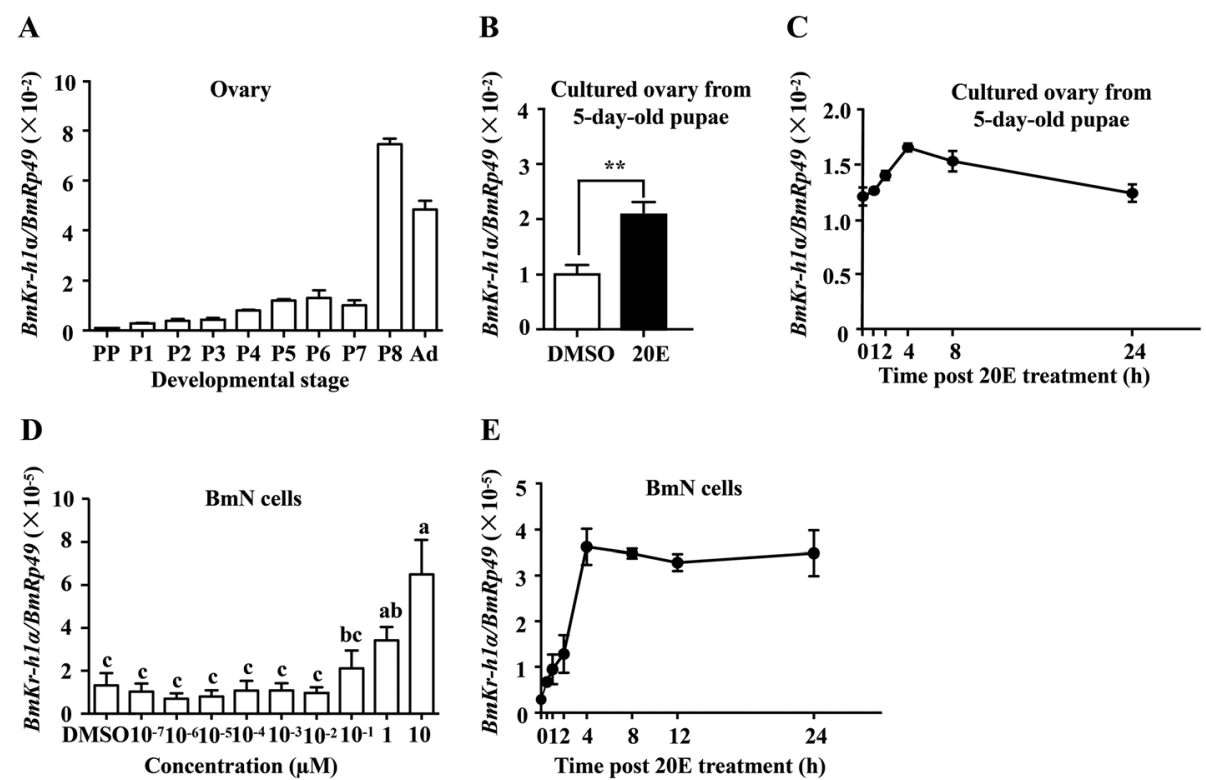

Fig. 1 Developmental and 20E-induced expression of BmKr-h1 in cultured ovaries and BmN cells. a Developmental expression profiles of BmKr-h1 in the ovaries. $\mathbf{b}, \mathbf{c}$ Induced expression and temporal changes in BmKr-hl at $2 \mathrm{~h}$ post $20 \mathrm{E}$ treatment $(1 \mu \mathrm{M})$ in the cultured ovaries from 5-day-old pupae. d, e Dose-dependent and time-dependent expression of BmKr-h1 expression in BmN cells. Data shown is mean $\pm \mathrm{SD}(n=3)$ and the individual data values are shown in Additional file 2. Different letters above the columns indicate significant differences at $p<0.05$ by ANOVA. The significance of the differences between the treatment and control was statistically analyzed at $p<0.05\left(^{*}\right), p<0.01(* *)$, and $p<0.001\left(^{* * *}\right)$ using $t$ test. $P$, pupal stage; PP, prepupal stage; Pn, day $n$ of pupal stage

the embryos inside the eggs died after oviposition (Additional file 1, Fig. S2), whereas the oocytes in the eggs injected with dsGFP were filled with normally developing embryos (Additional file 1, Fig. S2), results similar to that in our previous report [32]. Western blotting analysis showed that $\mathrm{Vg}$ levels were reduced in the ovaries of pupae on day 8 post ds $B m K r$ - $h 1$ treatment, compared to the control (Fig. 2C). Approximately $78 \%$ of the $\mathrm{ds} B m K r$ - $h 1$-treated adults laid eggs that eventually turned black, whereas only $15 \%$ of the dsGFP-treated adults did so. The hatch rate of the eggs laid by the $\mathrm{ds} B m K r$-h1-treated adults was reduced by approximately 7\% (Additional file, Table S1). However, the numbers of eggs laid by the females showed no obvious difference between the dsBmKr-h1-treated and dsGFP-treated adults (Additional file 1, Table S1). These results suggest that the suppression of $B m K r-h 1$ in late pupae inhibits the deposition of $\mathrm{Vg}$ in the ovary, thus preventing oocyte maturation.

To further determine how BmKr-h1 regulates Vg deposition, the expression of the $\mathrm{Vg}$ receptor $B m V g R$, which is responsible for the uptake of $\mathrm{Vg}$ into the oocytes, was examined in the oocytes of the ds $B m K r-h 1$ treated pupae. qRT-PCR analysis showed that silencing of $B m K r-h 1$ dramatically decreased the $B m V g R$ expression in the oocytes of dsBmKr-h1-treated pupae compared to the controls (Fig. 2D). Furthermore, the levels of $B m V g R$ and $B m V g$ were low in the ovaries during the early pupal stage, gradually increased during the midpupal stage, and then rapidly increased during the late pupal and adult stages (Additional file 1, Fig. S3A and $E)$, which was coincident with the expression patterns of $B m K r-h 1$ in the ovaries (Fig. 1a). The BmVgR expression in the cultured ovaries from 5-day-old pupae and BmN cells was also upregulated by $20 \mathrm{E}$ in a dose- and timedependent manner (Additional file 1, Fig. S3B and Fig. S3C-D). Furthermore, the BmVgR expression was increased when $B m K r-h 1$ was overexpressed in $B m N$ cells (Fig. 2E).

To determine whether the expression of $B m V g R$ was regulated directly by $B m K r-h 1$, the promoter of $B m V g R$ was searched for the presence of a potential $\mathrm{Kr}-\mathrm{h} 1$ binding site (KBS) sequence similar to the KBS homologous sequences in the promoters of the transcription factors $E 93$ and $B R-C[3,31]$. Three potential KBS sites $(-373 \sim-$ $362 \mathrm{nt},-2040 \sim-2027 \mathrm{nt}$, and $-2818 \sim-2805 \mathrm{nt})$ similar to the conserved motif GACCT were detected in the upstream region of the transcriptional start site of $B m V g R$ (Fig. 2F). Electrophoretic mobility shift assay (EMSA) analysis showed that the labeled $-2818 \sim-2805 \mathrm{nt}$ probe containing KBS specifically bound the nuclear proteins from BmKr-h1-EGFP overexpressing cells (Fig. 2G, lane $2-3)$, and 100-fold cold probe eliminated the binding (Fig. 2G, lane 4), whereas the labeled $-373 \sim-362$ nt and $-2040 \sim-2027 \mathrm{nt}$ probes did not bind nuclear proteins from BmKr-h1-EGFP overexpressing cells (Additional file, 


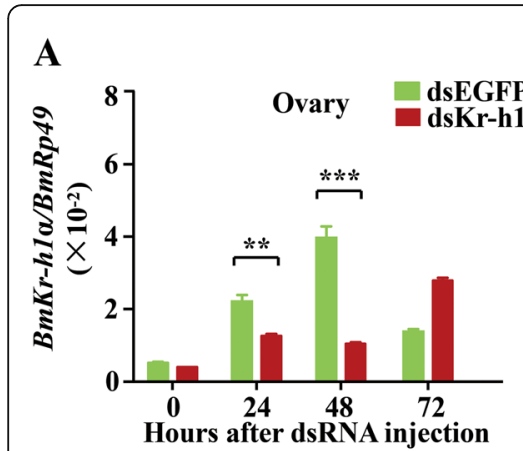

D
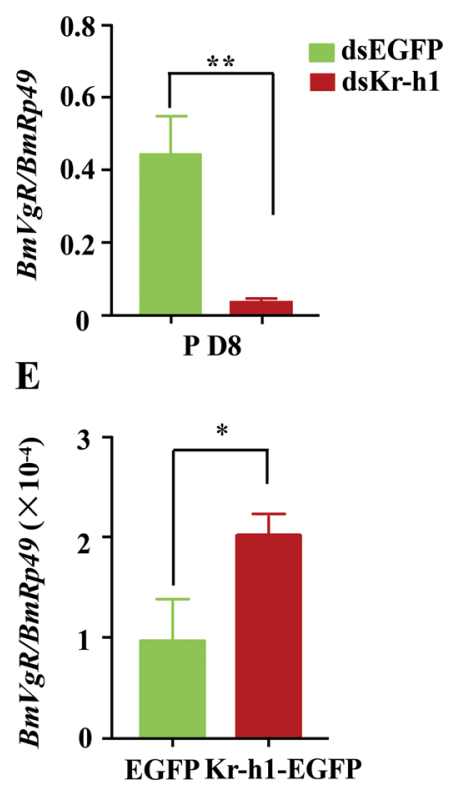

B

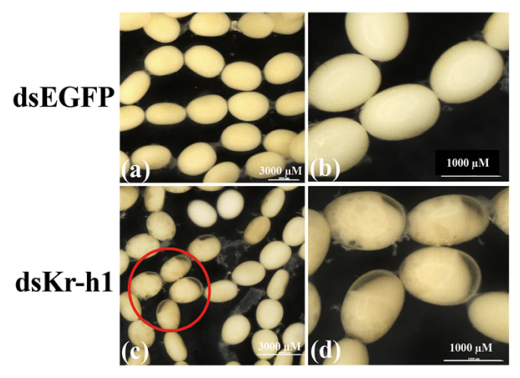

C

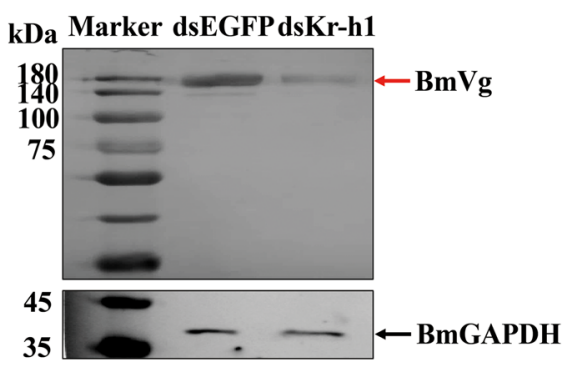

F

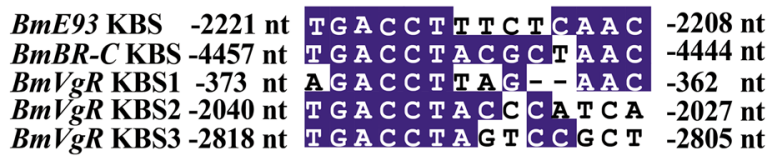

G

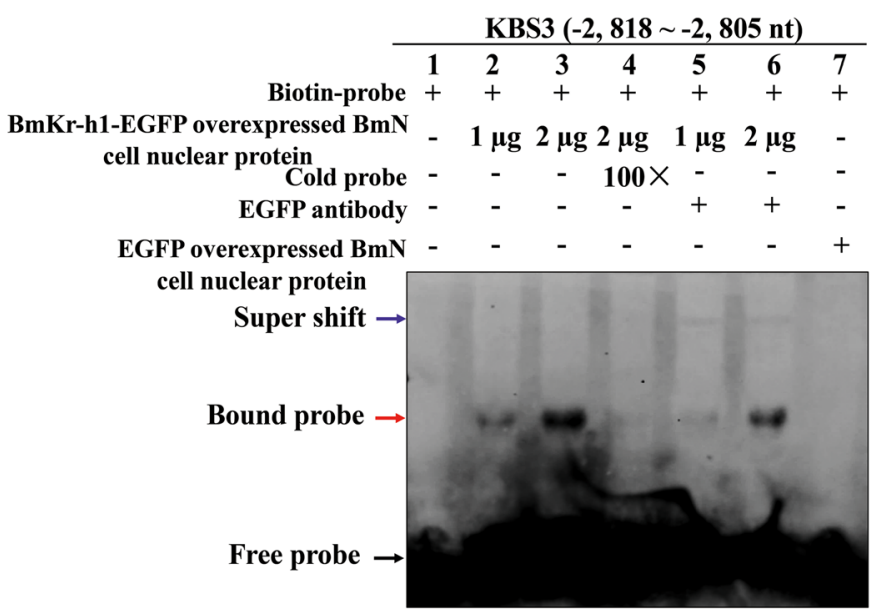

Fig. 2 Effects of RNAi mediated silencing of BmKr-h1 on the BmVgR expression and the oocyte development. A total of $10 \mu g$ of dsRNA per pupae was injected into the intersegmental region in the abdomen of 6-day-old pupae. A qRT-PCR analysis of BmKr-h1 mRNA in the ovaries of the treated pupae. B Phenotypes of oocytes in the ovaries of the dsRNA-treated pupae at day 8. C Western blot analysis of BmVg protein in the oocyte at $48 \mathrm{~h}$ post dsRNA treatment. A total of $15 \mu \mathrm{g}$ protein was loaded per lane and probed with anti-BmVg and anti-GAPDH antibodies, respectively. D qRT-PCR analysis of the expression change of $B m V g R$ in the oocyte. $\mathbf{E}$ qRT-PCR detection of the effect of BmKr-h1 overexpression on the transcript level of $B m V g R$. F Prediction of KBS in the BmVgR promoter based on the KBS core sequences of the BmBR-C and BmE93 promoters [3, 31]. The conserved amino acid residues are in blue background. G EMSA of the binding of the nuclear protein from BmN cells overexpressing BmKr-h1-EGFP with the KBS3 in the BmVgR promoter. For qRT-PCR, Rp49 amplified was used as the internal control. Significance of the differences between the treatment and control was statistically analyzed at $p<0.05\left(^{*}\right), p<0.01\left(^{* *}\right)$, and $p<0.001\left(^{(* *)}\right.$ using $t$ test. Dn, day of development; P, pupal stage

Fig. S4). When the BmKr-h1-EGFP-overexpressing nuclear proteins were incubated with the anti-EGFP polyclonal antibody, a weak supershifted binding signal was detected (Fig. 2G, lane 5-6), suggesting that the protein bound the KBS is BmKr-h1. These results suggest that BmKr-h1 directly binds the KBS $(-2818 \sim-2805 \mathrm{nt})$ in the promoter region of $B m V g R$ and upregulates its transcription, in turn enhancing Vg uptake by oocytes.

\section{Identification of 20E CREs in the BmKr-h1 promoter} To further explore the responsiveness of $B m K r-h 1$ to $20 \mathrm{E}$, the $20 \mathrm{E}$ cis-regulatory region $(20 \mathrm{CRE})$ in the
$B m K r-h 1$ promoter was identified using the dualluciferase reporter assays. Three regions of $B m K r-h 1$ promoter $(-2877 \sim+81 \mathrm{nt},-1877 \sim+81 \mathrm{nt}$, and -877 $\sim+81 \mathrm{nt}$ ) were cloned into pGL3-basic vectors and tested. Compared to pGL3-basic vector, all three constructs showed a significant activation of the luciferase reporter in the presence of $20 \mathrm{E}$, suggesting that the region between $-877 \sim+81 \mathrm{nt}$ is necessary for the $20 \mathrm{E}$ response (Fig. 3a). Thus, further truncated constructs within the $-877 \sim+81 \mathrm{nt}$ region $(-473 \sim+81 \mathrm{nt},-431$ $\sim+81 \mathrm{nt},-374 \sim+81 \mathrm{nt},-326 \sim+81 \mathrm{nt},-291 \sim+81$ nt, $-248 \sim+81 \mathrm{nt}$, and $-202 \sim+81 \mathrm{nt})$ were prepared 
and tested. The luciferase activity assays showed that the promoter activity of all these fragments was activated by 20E, except the shortest region, $-202 \sim+81 \mathrm{nt}$ (Fig. 3b), implying that the region between -248 and $-202 \mathrm{nt}$ is crucial for $20 \mathrm{E}$ response. To further confirm whether the $-248 \sim-202$ nt sequence of the $B m K r-h 1$ promoter is responsible for $20 \mathrm{E}$ induction, mutations were introduced in this region (TTATTAAAACATCCGAAA ACGCAATAATCATTCATGTAATTGAGAA were mutated to different nucleotides GGCGGCCCCACGAATC CCCATACCGCCGACGGACGTGCCGGTCTCC) and evaluated. The wild-type construct showed an activation in luciferase activity in the presence of $20 \mathrm{E}$, whereas the construct containing the mutant sequence of the region between -248 and $-202 \mathrm{nt}$ showed no response to $20 \mathrm{E}$ treatment (Fig. 3c). Thus, the region between -248 and - $202 \mathrm{nt}$ of the $B m K r-h 1$ promoter is demonstrated to be critical for 20E response.

To accurately pinpoint the nucleotide sequences within the $-248 \sim-202 \mathrm{nt}$ region required for response to 20E, EMSA was performed using biotin end-labeled double-stranded probes containing the $-248 \sim-202 \mathrm{nt}$ region and nuclear proteins extracted from 20E-treated $\mathrm{BmN}$ cells. Eleven probes, three for the $-234 \sim-202 \mathrm{nt}$ region and eight for the $-248 \sim-217 \mathrm{nt}$ region along with different mutations were prepared (Fig. 3d). BmN cell nuclear proteins were able to bind the wild-type probes and the binding was competed off by 50 -fold (Fig. 3e, lane 3) and 100-fold (Fig. 3f, lane 3) unlabeled probes. The binding signal was weakened when the mutant probe 1 (TCCCCATACCGCCGACGGCATGTAAT TGAGAA, the nucleotides between -234 and $-217 \mathrm{nt}$ were mutated in the $234 \sim-202 \mathrm{nt}$ region) was used. However, the binding was still detected for mutant probe 2 (GAAAACGCAATAATCATTACGTGCCGGT CTCC, the nucleotides between -217 and -202 nt were mutated in the -234 to -202 nt region (Fig. 3e, lane 45), suggesting that the region (GAAAACGCAATAAT CATT) is essential for binding, whereas the region (CATGTAATTGAGAA) is not. In addition, the binding signal was weakened when the mutant probes 1,2 , and 3 of the $-248 \sim-217 \mathrm{nt}$ region were used (Fig. 3f, lane 46), whereas the mutant probe 4 did not alter the binding signal (Fig. 3e, lane 7), suggesting that the region (AACA TCCGAAAACGC) is not critical for the binding. We then mutated the flanking sequences of the probe $-248 \sim-$ $217 \mathrm{nt}$ region individually or simultaneously (Fig. 3d). When the left and right flanking sequences of the -248 $-217 \mathrm{nt}$ region were mutated simultaneously as shown in the mutant probe 5, the binding signal disappeared (Fig. 3f,

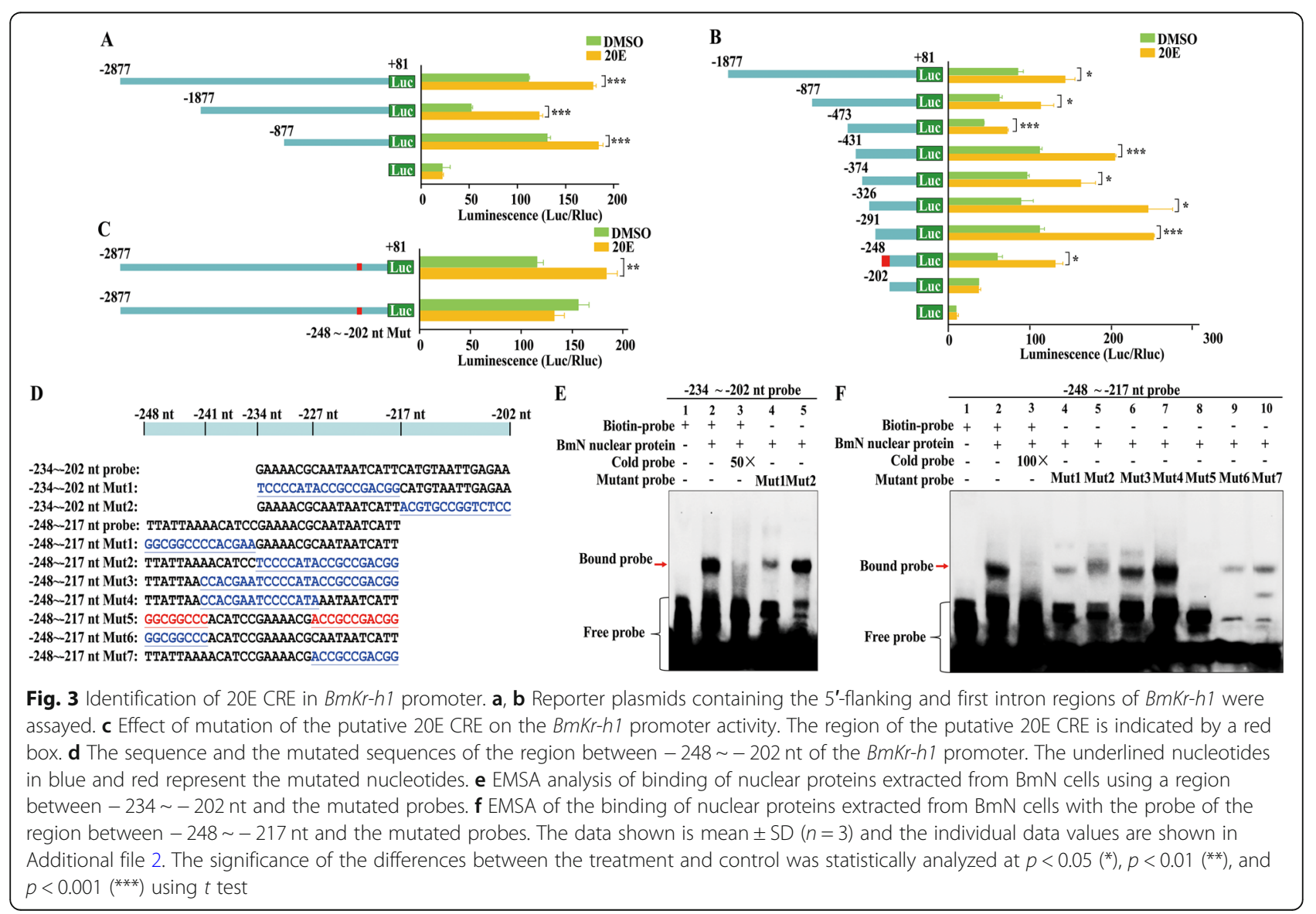


lane 8). However, when only the left sequence (the mutant probe 6) or right sequence (the mutant probe 7) was mutated individually, the binding signal was weakened but not completely abolished (Fig. 3f, lane 9-10). These results together suggest that the essential protein-binding sites (or 20E CRE) of the $B m K r-h 1$ gene are located at - 248 - $241 \mathrm{nt}$ (TTATTAA) and - $227 \sim-217$ nt (AATAATCA TT) in the promoter.

We then screened public genomic databases for the sequences homologous to the potential 20E CRE in other insect species. Sequences with similarity to the 20E CRE (TTATTAA or AATAATCATT) were found in the $3-\mathrm{kb}$ upstream regions of $K r-h 1$ in T. castaneum, Apis mellifera, A. aegypti, Acyrthosiphon pisum, and D. melanogaster. All these species possess identical TTATTAA sequences (Additional file 1, Fig. S5A) and AATAATCATT sequences (Additional file 1, Fig. S5B) in their upstream regions. Moreover, like $B m K r$ - $h 1$, the AATAATCATT sequence was found adjacent to or partially overlapping with the TTATTAA sequence in their $K r-h 1$ promoters in all these species (Additional file 1, Fig. S5).

\section{Identification of 20E CRE binding protein}

To identify the nuclear protein(s) that bind the 20E CRE ($248 \sim-217 \mathrm{nt})$ in the BmKr-h1 promoter, a DNA pull-down assay was performed. The biotin-labeled CRE containing nucleotide fragment (TTATTAAAACATCCGAAAACGCAA TAATCATT) was linked to the streptavidin-coated beads and incubated with nuclear proteins isolated from 20Etreated BmN cells. A mutated probe (GGCGGCCCACATC CGAAAACGACCGCCGACGG) that does not bind the nuclear proteins was used as a control. The protein(s) binding the probe were then separated on an SDS-PAGE gel, and the protein bands present in the purified proteins using the wildtype CRE but not in those purified with the mutant CRE (Fig. 4A) were excised and subjected to LC-MS/MS analysis. LC-MS/MS analysis identified fifteen candidate proteins (Additional file 1, Table S2). One of these proteins, BmLOC101738779 (GenBank accession no. XM 004922171.3) is a nucleic acid-binding and potential 20E CRE-binding protein and hence named BmKRP (B. mori Kr$h 1$ regulatory protein). This protein consists of 518 amino acids with a predicted molecular mass of $59 \mathrm{kDa}$ and a $\mathrm{pI}$ of 9.25, containing three $\mathrm{ZnF}-\mathrm{C}_{2} \mathrm{H}_{2}$ domains at its $\mathrm{N}$-terminal end and three low-complexity regions at the $C$-terminal end (Fig. 4B and Additional file 1, Fig. S6).

To examine subcellular localization of BmKRP, expression vectors of BmKRP-EGFP and EGFP (as a control) were constructed and used to transfect $\mathrm{BmN}$ cells. The localization of the expressed proteins was examined under a confocal microscope. The green fluorescence signal in the cells transfected with EGFP alone was present in both the nuclei and cytoplasm (Fig. $4 \mathrm{C} \mathrm{a-C}$ ), whereas the BmKRP-EGFP signal was exclusively localized to the nuclei (Fig. $4 \mathrm{C} \mathrm{d}-\mathrm{f}$ ), suggesting that this protein may be involved in the regulation of $B m K r-h 1$ transcription in the nucleus.

To determine whether this protein binds the 20E CRE in the $B m K r-h 1$ promoter, the protein was translated in vitro and used in EMSA. BmKRP specifically bound the labeled $-248 \sim-217 \mathrm{nt}$ probe (Fig. 4D, lane 2 and 8 ), and the binding was competed off by the 50 -fold excess unlabeled probe (Fig. 4D, lane 3). No such binding was found when the protein was translated from control vector DNA (Fig. 4D, lane 9). The BmKRP protein bound the mutated probes 4, 6, and 7 (Fig. 4D, lane 4, 6, and 7), but did not bind the mutated probe 5 (Fig. 4D, lane 5). These results indicate that the binding of the recombinant protein with the CRE is consistent with that of the nuclear proteins from BmN cells (Fig. 3f).

To further demonstrate whether BmKRP bound the 20E CRE in the BmKr-h1 promoter in vivo, a ChIP assay was performed. BmKRP was expressed as an EGFP fusion protein and the antibodies that recognize EGFP were used in ChIP assay. BmN cells were transfected with BmKRP-EGFP or EGFP (as a control) for $48 \mathrm{~h}$. The cells were then collected and processed for ChIP assay. The EGFP antibodies, but not IgG (negative control), precipitated and enriched the 20E CRE fragment of the $B m K r-h 1$ promoter in the cells transfected with the BmKRP-EGFP-expressing plasmid (Fig. 4E). The PCRenriched 20E CRE sequence was confirmed by sequencing (Fig. 4F, G). These experiments demonstrated that BmKRP, which was localized to the nucleus (Fig. $4 \mathrm{C} \mathrm{d-f),}$ binds the 20E CRE located in the $-248 \sim-217 \mathrm{nt}$ region of the $B m K r-h 1$ promoter.

To determine whether the binding of BmKRP to the 20E CRE influenced the $B m K r-h 1$ promoter transcription activity, the BmKRP-EGFP expression vector was co-transfected into BmN cells with the $B m K r$ - $h 1$-Promoter $(-248 /+81 \mathrm{bp})-\mathrm{Luc}$ construct. The BmKr-h1 promoter-controlled luciferase activity increased in the BmKRP-EGFP-transfected cells but not in the control cells (Fig. 4H). To confirm the BmKRP overexpression result, BmKRP RNAi was performed. When the BmKRP expression was downregulated by dsBmKRP in $\mathrm{BmN}$ cells (Fig. 4I), the transcription activity of the wild-type $B m K r-h 1$ promoter induced by $20 \mathrm{E}$ disappeared compared to the control cells treated with dsEGFP (Fig. 4J). The transcription activity of the mutated $B m K r-h 1$ promoter did not respond to $20 \mathrm{E}$ challenge in either dsBmKRP- or dsEGFP-treated cells (Fig. 4J).

The developmental and 20E-induced expression patterns of $B m K R P$ were then examined. qRT-PCR analysis showed that in the ovaries, the BmKRP mRNA levels were low during the early- and mid-pupal stages, but dramatically increased during the late pupal and adult stages (Additional file 1, Fig. S7A). Treatment with 20E 


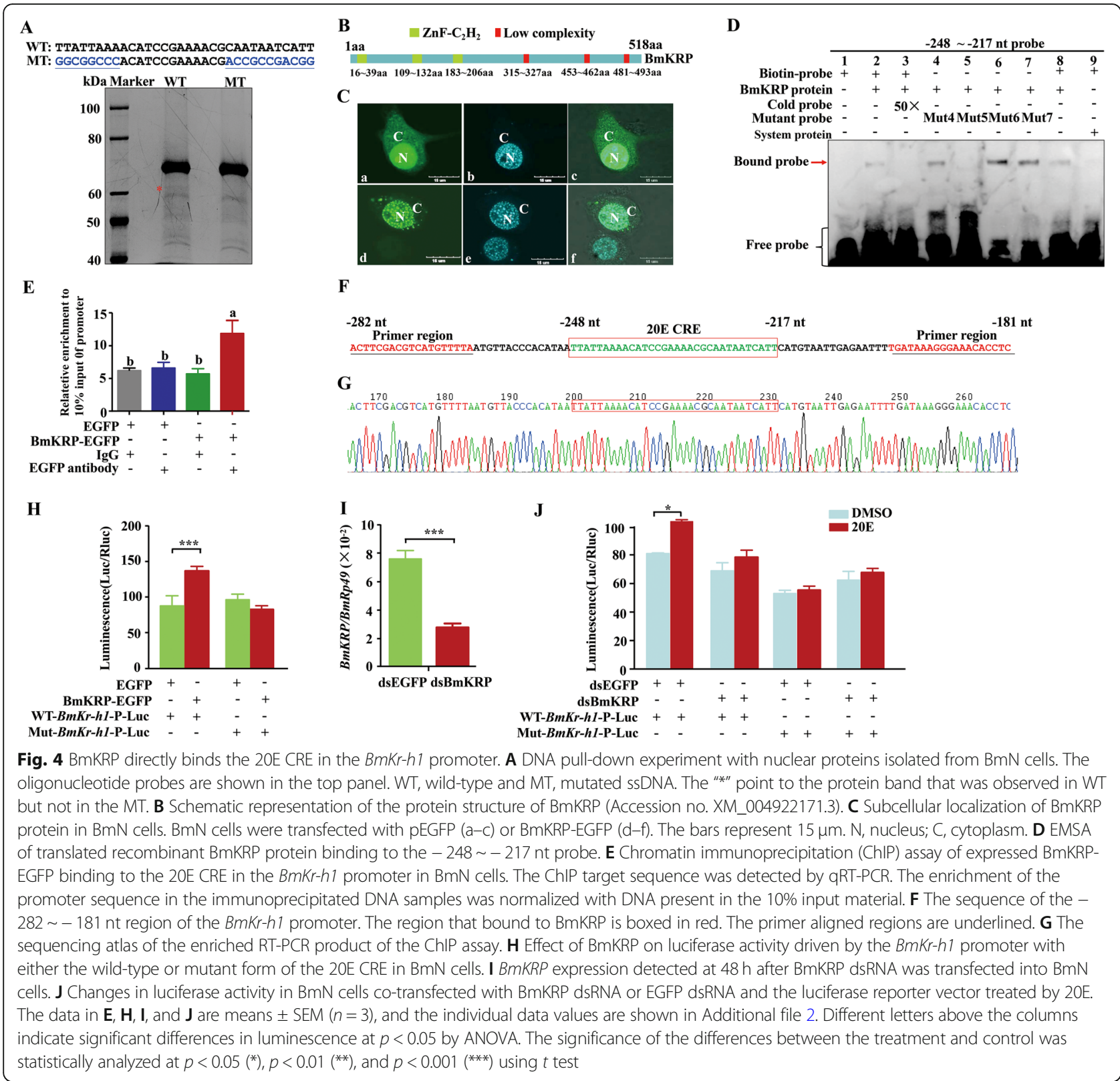

increased the expression of $B m K R P$ in the cultured ovaries dissected from 5-day-old pupae and in $\mathrm{BmN}$ cells (Additional file 1, Fig. S7B-D). Additionally, a potential EcRE (-1717 - $1705 \mathrm{nt}$, AGTTCAGTGACCT) was predicted in the promoter of $B m K R P$ (Additional file 1, Fig. S7E) based on the consensus EcRE sequence (5'-(A/ G)G(G/T)TCANTGA(C/A)C(C/T)-3') for 20E/EcR/USP binding [33]. These results indicate that 20E induces the $B m K R P$ expression by binding the EcR/USP complex which in turn interacts with the potential EcRE in the $B m K R P$ promoter. BmKRP then binds the 20E CRE in the $B m K r-h 1$ promoter and activated its expression in the ovaries and BmN cells.

\section{Effects of BmKRP knockout on ovarian development and oogenesis}

To determine whether BmKr-h1 promotes the oocyte development through BmKRP, the mutant $B$. mori line for Bmkrp was established using the CRISPR/Cas9-mediated genome editing. A 23-bp sgRNA target site located on exon 1 was identified by screening for sgRNA sites over the exons of BmKRP (Fig. 5A). The synthesized sgRNA was mixed with Cas9 mRNA and injected into preblastoderm embryos of the silkworm. In the third generation, the homozygous individuals were obtained and two mutant genotypes (mutant $1,4 \mathrm{bp}$ deletion; mutant2, $2 \mathrm{bp}$ deletion) were identified (Fig. 5B). The 
$\mathrm{Bmkrp}^{-/-}$pupae and adults, especially females, were smaller than the wild-type (WT) animals, while the $B m k r p^{-1-}$ larvae were not obviously different in body size and shape from the wildtype (Additional file 1, Fig. S8B). Compared to the wild-type, the body weight, length, and width of the $B m k r p p^{-/-}$pupae at day 4 was lower (Additional file 1, Fig. S8C-E). The development of the ovary was incomplete and contained fewer oocytes in the $\mathrm{Bmkrp}^{-/-}$pupae and adults than that in the wildtype (Fig. $5 \mathrm{C}$ ). The number of eggs laid by the mutant females was reduced by $46 \%$ (Additional file 1, Fig. S8FG). In addition, the length and width of oocytes in the ovarioles were reduced (Fig. 5C (b), C (e), and D). Furthermore, western blotting analysis showed that the major yolk protein $\mathrm{Vg}$ was reduced in the oocytes of 7day-old pupae and 1-day-old adults, as compared to the wild-type (Fig. 5E). This is similar to the reduction of $\mathrm{Vg}$ in the oocytes of dsBmKr-h1-treated females (Fig. 2C). These results suggest that the depletion of $B m K R P$ reduces Vg accumulation, perturbs ovarian development and arrests oogenesis.

To confirm whether $B m K R P$ regulated the ovarian development and oocyte formation via $B m K r-h 1$ and $B m V g R$, the expression of $B m K r-h 1$ and $B m V g R$ in the oocytes of the $B m k r p^{-1-}$ silkworm were quantified. qRTPCR analysis showed that the expression of $B m K r-h 1$ and $B m V g R$ decreased in the oocytes of $B m k^{2} p^{-1-}$ pupae collected on day 7 compared to the wild-type (Fig. 5F, G), suggesting that BmKRP is a regulator of $B m K r-h 1$ and $B m V g R$.

\section{Discussion}

The data presented in the present paper form lines of evidence supporting our hypothesis that 20E-induced $\mathrm{Kr}$-h1 expression is mediated by a novel transcription factor BmKRP in the female reproductive system of a model lepidopteran insect $B$. mori. Our data show the following conclusions: (1) BmKr-h1 was highly expressed in ovaries during the late pupal and adult stages when $20 \mathrm{E}$ titers were high and was experimentally demonstrated to be induced by 20E; (2) dsRNA knockdown of $B m K r-h 1$ led to arrested oogenesis via inhibiting the expression of $B m V g R$; (3) A 20E CRE was identified in the promoter of $B m K r-h 1$ and functionally verified using luciferase reporter assay, EMSA and DNA-ChIP; (4) A novel transcription factor BmKRP that specifically bound the 20E CRE of $B m K r-h 1$ promoter was shown to activate $B m K r$ - $h 1$ transcription; and (5) CRISPR/Cas9-mediated knockout of $B m K R P$ in female pupae suppressed the transcription of $B m K r-h 1$ and $B m V g R$, resulting in arrested oogenesis. These data suggest that the 20Einduced BmKRP regulates BmKr-h1 expression, which in turn activates $\mathrm{VgR}$ expression, facilitating $\mathrm{Vg}$ uptake and oogenesis.
Induction of $K r-h 1$ gene by both $\mathrm{JH}$ and 20E has been reported in different species [16-20, 28]. The mechanisms and physiological functions of $\mathrm{JH}$-induced $\mathrm{Kr}-\mathrm{h} 1$ have been well studied $[15,28]$. Recent studies report that $K r$ - $h 1$ transduces the JH signal to suppress $20 \mathrm{E}$ primary responsive genes, such as ecdysone receptor $(E c R)$, $B R-C$, and ecdysone-inducible proteins E75 and E93, which subsequently lead to a lowering of $20 \mathrm{E}$ titer [34], and $\mathrm{Kr}$-h1 inhibits the expression of steroidogenic enzyme gene Spok by binding the Kr-h1 binding site (KBS) to suppress the ecdysone biosynthesis [35], partially explaining the sophisticated JH-20E cross-talk. However, the physiological role and regulatory mechanism of the 20E-induced $K r$ - $h 1$ expression is not known. We investigated the physiological role and mechanisms of 20Einduced $K r-h 1$ in female reproduction of $B$. mori. The developmental and 20E-induced expression studies showed that $B m K r-h 1$ is induced by $20 \mathrm{E}$ in the cultured ovaries of 5-day-old pupae and BmN cells of B. mori (Fig. 1), suggesting a role for the 20E-induced BmKr-h1. In B. mori, 20E-dependent oogenesis occurs almost exclusively during pupal and pharate adult stages when $\mathrm{JH}$ is absent and is completed before adult eclosion [36]. A high ecdysone titer was detected in the hemolymph of 3day-old pupae [29] and in the ovaries of 7-day-old pupae in B. mori [30]. RNAi-mediated silencing of $B m K r-h 1$ in late female pupae downregulated the expression of $B m V g R$ and reduced the deposition of $\mathrm{Vg}$ protein into the oocytes (Fig. 2), suggesting that BmKr-h1 regulates the uptake of yolk protein through BmVgR.

$\mathrm{Vg}$ synthesis and ovarian development are coregulated by a complex interaction between $20 \mathrm{E}, \mathrm{JH}$ and nutritional signaling pathway, in which $K r-h 1$ plays critical roles [37]. In $H$. armigera, HaKr-h1 RNAi results in atrophied ovaries with less yolk protein deposition [19]. In L. migratoria, $N$. lugens, $A$. aegypti, $H$. armigera, $B$. dorsalis and $S$. furcifera, depletion of $K r-h 1$ prevents JH-regulated expression of $\mathrm{Vg}$ and oocyte maturation, ovarian development and egg production [19, 21-25]. Knocking down $K r-h 1$ in female adults of $C$. lectularius does not reduce their fecundity but affects the embryonic development [27]. In $N$. lugens, silencing of both Broad-complex and $\mathrm{Kr}$ $h 1$ increases the number of eggs laid by long-winged females, whereas knocking down either one of these two genes decreases the number of eggs laid [38]. The inhibitory effect of knocking down E93 on ovarian development and egg number is partially recovered by $K r-h 1$ knockdown [39]. Thus, either $\mathrm{JH}$ or $20 \mathrm{E}$ regulates $K r-h 1$ expression, vitellogenesis and ovarian development in different insect species. These differences may be due to differential hormonal regulation of oogenesis and the various types of ovaries. For instance, JH regulates the reproduction in 


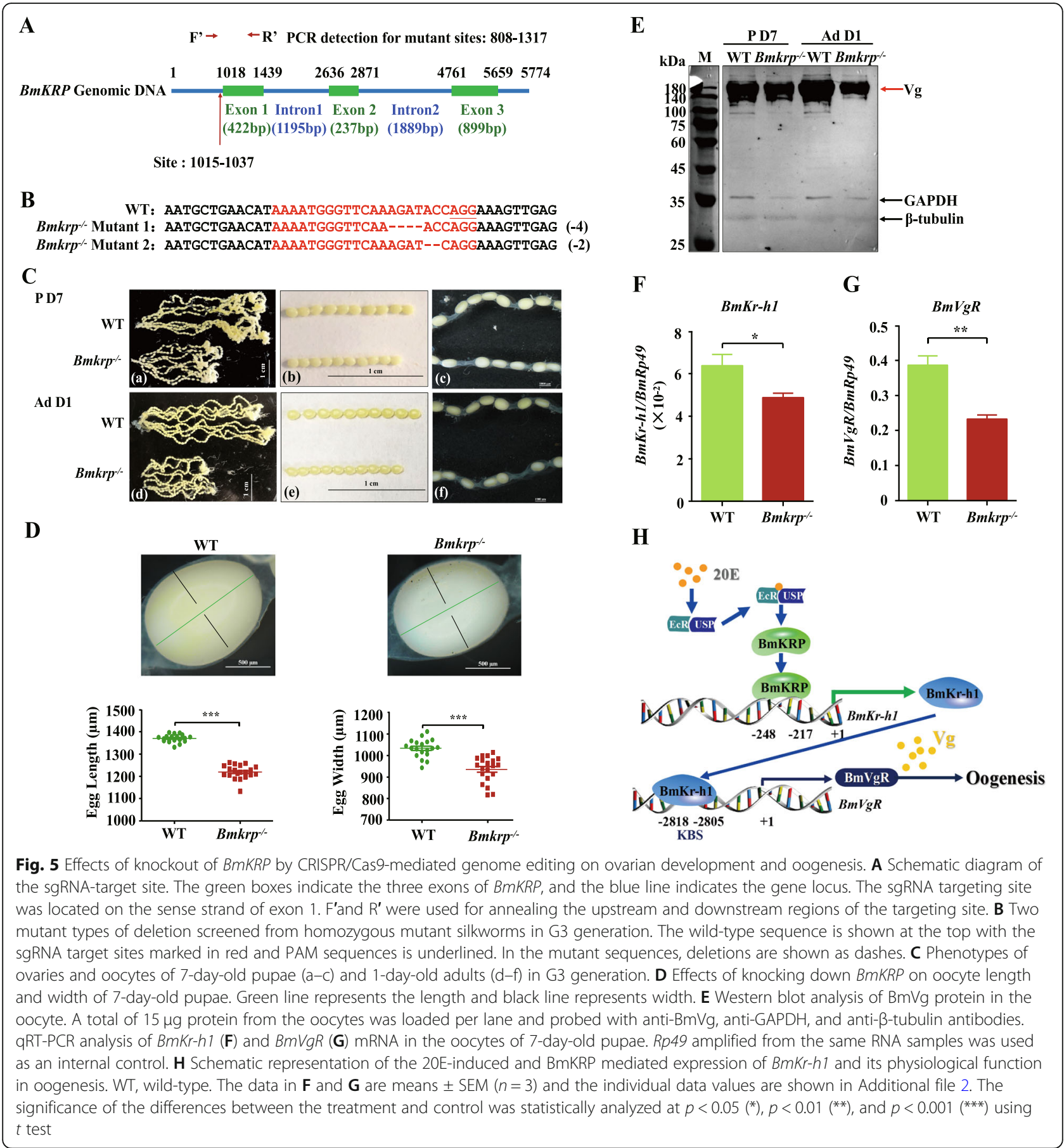

hemimetabolous insect orders, such as Orthoptera ( $L$. migratoria) [21, 40, 41], Blattodea (Blattella germanica, Diploptera punctata) [42-44] and Hemiptera (Pyrrhocoris apterus, C. lectularius, N. lugens) [14, 22, 45]. In holometabolous coleopteran T. castaneum, JH is the primary hormone governing reproduction [26], but ecdysteroids are involved in oocyte maturation [46]. In lepidopterans, reproduction regulation appears to be quite different. $\mathrm{JH}$ has a significant role in many species, including Manduca sexta and $H$. armigera, but in others, such as B. mori, ecdysteroids are the major hormone controlling egg development [37]. In the fall armyworm Spodoptera frugiperda, ecdysteroids stimulate vitellogenesis, and $\mathrm{JH}$ promotes $\mathrm{Vg}$ uptake into the ovaries [47]. These results indicate the complexity in the hormonal regulation of the 
reproductive process in insects and both $\mathrm{JH}$ and $20 \mathrm{E}$ may contribute to different steps of the process.

The present study found that $\mathrm{BmKr}-\mathrm{h} 1$ regulated the expression of $B m V g R$ by directly binding its promoter, enhancing Vg uptake into oocytes. Depletion of either $B m K R P$ or $B m K r-h 1$ suppressed the $B m V g R$ expression in late female pupae (Figs. 2D and $5 \mathrm{G}$ ), and overexpression of $B m K r-h 1$ increased the expression of $B m V g R$ in $B m N$ cells (Fig. 2E). BmKr-h1 specifically bound the KBS located at the $-2818 \sim-2805 \mathrm{nt}$ upstream regulatory region of $B m V g R$ (Fig. 2G). $K r-h 1$ had been reported to contribute to an anti-metamorphic function of $\mathrm{JH}$ by binding to the KBS sequences of $E 93, B R-C$ and steroidogenic enzyme genes [3, 31, 34, 35]. Although $K r-h 1$ had been found to be involved in female reproduction, the detailed molecular regulatory mechanism had not been clearly understood. Here, we reported the identification of the $K r$ - $h 1$ target gene, $B m V g R$, and the molecular mechanism of $K r$-h1 function in female reproduction. $B m K r-h 1$ upregulated the $B m V g R$ expression by binding to the KBS in its promoter, enhancing the $\mathrm{Vg}$ uptake by oocytes and egg maturation. It must be noted that we could not exclude the possibility that other genes involved in the oogenesis might also be regulated by BmKr-h1 and further investigation is needed.

Because $B m K r-h 1$ showed a rapid response to $20 \mathrm{E}$, a 20E CRE in the upstream regulatory region of the gene was expected. The $-248 \sim-217$ nt region of the BmKr-h1 gene was identified as the 20E CRE region (Fig. 3), the sequence of which differed from EcRE identified in 20Eresponse genes [33]. In particular, the sequences at -248 $\sim-241 \mathrm{nt}$ (TTATTAA) and $-227 \sim-217 \mathrm{nt}$ (AATAAT CATT) regions, separated by $13 \mathrm{bp}$, are required for the 20E response (Fig. 3d, f). When either one of the CREs was mutated (Mut 6 and Mut 7), the binding with BmKRP still occurred although the binding became weaker (Fig. 3f, lane 9 and 10). Similar 20E CREs, TTATTAA and AATA ATCATT, which are adjacent to each other or partially overlapping, were also found in the upstream region of $K r$-h1 genes in T. castaneum, A. mellifera, A. aegypti, A. pisum and D. melanogaster (Additional file 1, Fig. S5), indicating that the 20E CREs of $K r-h 1$ are conserved across different species. The EcRE upstream of $B m E c R-B 1$ is also composed of two adjacent elements (E1 and E2) with $5 \mathrm{bp}$ separation, and both are required for the 20E response and the two-component structure of E1 and E2 in EcR-B1 was conserved in lepidopteran species [48]. The above results suggest that two adjacent 20E CRE in the upstream of a gene may enable the rapid induction of gene transcription by $20 \mathrm{E}$, which probably is a common mechanism in the $20 \mathrm{E}$ signaling pathway.

The relationship between the $\mathrm{JH}$ response element (JHRE) and 20E CRE in the promoter region of $\mathrm{BmKr}$ $h 1$ is an interesting subject. The two 20E CREs are located at $-248 \sim-241 \mathrm{nt}$ and $-227 \sim-217 \mathrm{nt}$ regions, while the JHRE in the $B m K r-h 1$ promoter is located at the $-2165 \sim-2025 \mathrm{nt}$ region [28]. These two 20E CREs and JHRE are separated by about 1917 bps in the $B m K r-$ $h 1$ promoter. How they interact or cooperate to regulate the $B m K r-h 1$ expression is not known yet. They might function separately or simultaneously in different tissues and stages so that $B m K r-h 1$ could differentially respond to $\mathrm{JH}$ and $20 \mathrm{E}$ in the regulation of molting, metamorphosis and reproduction. This is an interesting issue that should be further investigated in a future study.

Identification of proteins that participate in the regulation of $B m K r-h 1$ by binding the CRE is an important objective of this study. A $B m K r-h 1$ regulatory protein, BmKRP, was identified and demonstrated to be able to bind the CREs in the $B m K r-h 1$ promoter (Fig. 4). BmKRP contains three $\mathrm{ZnF}-\mathrm{C}_{2} \mathrm{H}_{2}$ domains at the $\mathrm{N}$-terminal end and is localized in the nuclei of $\mathrm{BmN}$ cells (Additional file 1, Fig. S6 and Fig. 4B, C). BmKRP had high expression levels in the ovaries during the latepupal and adult stages and was induced by $20 \mathrm{E}$ in the cultured ovaries and ovarian $\mathrm{BmN}$ cells (Additional file 1, Fig. S7), as was $B m K r-h 1$ (Fig. 1) and $B m V g R$ (Additional file 1, Fig. S3A-D). Overexpressing BmKRP enhanced the promoter activity of $B m K r-h 1$, whereas knocking-down BmKRP with RNAi decreased the promoter activity of $B m K r-h 1$ in BmN cells (Fig. $4 \mathrm{H}-\mathrm{J}$ ). This evidence suggests that BmKRP in responding to $20 \mathrm{E}$ mediates $B m K r-h 1$ expression by binding its $20 \mathrm{E}$ CRE. It would be interesting to know how BmKRP, which has three $\mathrm{ZnF}-\mathrm{C}_{2} \mathrm{H}_{2}$ domains, structurally bind the two CREs in the $B m K r$ - $h 1$ promoter. In addition, knocking out $B m K R P$ by CRISPR/Cas9 method also reduced the expression of $B m K r-h 1$ and $B m V g R$ (Fig. 5F, G) and Vg deposition in the oocytes (Fig. 5E), resulting in arrested development in ovaries and oogenesis (Fig. 5C, D) that was also observed in the dsBmKr-h1treated females (Fig. 2). These results suggest that the 20E-induced $B m K r-h 1$ expression is mediated by BmKRP and promotes oocyte development through $B m V g R$.

In summary, in the ovaries of late pupae, 20E induced the expression of $B m K R P$, which activated the $B m K r-h 1$ expression by interacting with the 20E CREs in its promoter. Then, $B m K r-h 1$ bound the KBS in the promoter of $B m V g R$ to activate its expression. $B m V g R$ facilitated the BmVg uptake by the oocytes, resulting in oocyte maturation (Fig. $5 \mathrm{H}$ ). In this model, $B m K r-h 1$ is a critical player between the $20 \mathrm{E}$ signal and oogenesis.

\section{Conclusion}

The induced expression of $K r-h 1$ by $20 \mathrm{E}$ exists in a variety of insects. $B m K r-h 1$ was highly expressed in ovaries 
during the late pupal and adult stages and the expression was induced by 20E. RNAi-mediated Depletion of $B m K r-h 1$ in female pupae during the late pupal stage repressed the transcription of vitellogenin receptor $(V g R)$ and reduced the $\mathrm{Vg}$ deposition in oocytes. Then, a $20 \mathrm{E}$ CRE in the promoter region of $B m K r-h 1$ and a novel $\mathrm{Kr}$ $h 1$ regulatory protein $(\mathrm{BmKRP})$ that binds this CRE were identified. Furthermore, in responding to $20 \mathrm{E}$ stimulation, BmKRP was shown to induce $B m K r-h 1$ expression, which in turn induced $B m V g R$ expression to facilitate $V g$ uptake and oogenesis. Our data demonstrated that 20Einduced BmKRP mediated $K r-h 1$ expression in the female reproduction of $B$. mori.

\section{Methods}

\section{Insects, cells, and treatments}

The silkworm B. mori strain P50 was provided by the Research and Development Center of the Sericultural Research Institute of the Academy of Agricultural Sciences of Guangdong Province, China. Larvae were reared in incubators at $25-27^{\circ} \mathrm{C}$ under a photoperiod of $12 \mathrm{~h}$ light and $12 \mathrm{~h}$ dark and fed with fresh mulberry leaves. Under these conditions, larvae wandered on day 6 or 7 of the 5 th instar and pupated 3 days thereafter. The pupal stage was 8 days and then emerged to adults. P0 was the moment at which larvae just shed the remnants of the larval integument, marking the beginning of the pupal stage. The ovaries in 5-day-old pupae were prepared for in vitro culture. The ovaries and oocytes were dissected, rinsed with phosphate-buffered saline (PBS), and cultured in Grace's medium (Gibco, Grand Island, NY, USA). A BmN (B. mori ovaries derived) cell line was maintained at $28{ }^{\circ} \mathrm{C}$ in Grace's Insect Medium (Gibco, Grand Island, NY, USA) and with 10\% FBS (Gibco, Grand Island, NY, USA).

The cultured ovaries and BmN cells were treated with different concentrations of 20E (Sigma Co., St. Louis, MO, USA) and the ovaries or cells were collected after $4 \mathrm{~h}$ in culture. In the time-course experiment, the cultured ovaries or BmN cells were treated with $1 \mu \mathrm{M} 20 \mathrm{E}$ and collected in different time points. All the samples were immediately frozen with liquid nitrogen at the end of culture and stored at $-80^{\circ} \mathrm{C}$ until use for RNA extraction.

RNA extraction and quantitation real-time PCR (qRT-PCR) Total RNA was extracted from the collected ovaries, the cultured ovaries, oocytes or the $\mathrm{BmN}$ cells and firststrand cDNA was synthesized according to the instructions (TaKaRa Co. Dalian, China). The primers used were shown in Table S3 (Additional file 1). BmKr-h1 expression levels were determined by qRT-PCR using SYBR Select Master Mix (Applied Biosystems, Austin, USA) according to the instructions and were calculated by the comparative $\mathrm{Ct}\left(2^{-\Delta \Delta \mathrm{Ct}}\right)$ method [49]. All the experiments were performed for three replicates.

BmKr-h1 promoter cloning and site-directed mutagenesis The upstream regulatory region (from $-2877 \mathrm{bp}$ to +81 $\mathrm{bp)}$ of $B m K r-h 1$ was amplified based on the sequence from NCBI (GenBank accession no.: BAJ05087) and cloned into pMD-18 T vector (TaKaRa Co., Dalian, China). A series of stepwise truncated fragments of the $B m K r-h 1$ regulatory region starting at position $+81 \mathrm{bp}$ and extending to $-1877,-877,-473,-431,-374,-326$, $-291,-248$, and -202 bp were generated by PCR amplification using the $B m K r-h 1$-Promoter $(-2877 /+81 \mathrm{bp})$ plasmid as a template and ligated to the luciferase reporter plasmid, pGL3-basic vector (Promega, Madison, WI, USA). The sequences of the forward primers and a common reverse primer are listed in Supplementary Table S3. The mutant (mutated sites from -248 to $-202 \mathrm{nt}$ ) BmKr-h1-Promoter $(-2877 /+81 \mathrm{bp})$ were generated with the method of overlap extension PCR using the wild-type $B m K r-h 1$-Promoter (-2877/+ $81 \mathrm{bp}$ ) plasmid as a template. The mutant was confirmed by DNA sequencing and then inserted to pGL3-basic vector for activity assay.

\section{Luciferase assay for promoter activity}

Cell transfection and co-transfection were conducted when the cell density achieved $80 \%$. To normalize the firefly luciferase activity, the renilla luciferase vector pRL-SV40 (Promega, Madison, USA) was co-transfected with each of the pGL3-derived vectors. For transfection, a mix of $50 \mu \mathrm{L}$ containing $500 \mathrm{ng}$ of the wild-type or mutated $B m K r-h 1$ promoter-pGL3 reporter plasmid DNA, $50 \mathrm{ng}$ internal control plasmid and $1.5 \mu \mathrm{L}$ Fugene HD transfection reagent (Promega, Madison, USA) in the Opti-MEM reduced serum medium (Invitrogen, CA, USA) was added to BmN cells in Grace medium with $0.005 \%$ DMSO (final concentration) or $1 \mu \mathrm{M} 20 \mathrm{E}$ in $0.005 \%$ DMSO. For co-transfection, a mix of $50 \mu \mathrm{L}$ containing $500 \mathrm{ng}$ of the wild-type or mutated $B m K r-h 1$ promoter-pGL3 reporter plasmid DNA, 500 ng EGFP or BmKRP-EGFP plasmid DNA, $100 \mathrm{ng}$ internal control plasmid (pRL-SV40 vector) and $3 \mu \mathrm{L}$ Fugene HD transfection reagent in the Opti-MEM Reduced Serum Medium was added to the cells. The cells were cultured for $48 \mathrm{~h}$ at $28^{\circ} \mathrm{C}$ before the promoter activity assay. The luciferase activity assay was carried out according to the instruction of the Dual-Luciferase ${ }^{\circledast}$ Reporter Assay System Kit (Promega, Madison, USA). All assays were repeated at least three times. The luciferase activity was represented as mean \pm standard error (SE). Statistical significance of the luciferase activity was analyzed using the Student's $t$ test. 
DNA pull-down and liquid chromatography-tandem mass spectrometry (LC-MS/MS) analysis

Nuclear protein of BmN cells was extracted using NEPER Nuclear and Cytoplasmic Extraction Reagents (Thermo Scientific, Waltham, USA). The protein concentration was determined using BCA Protein Assay Reagent Kit (Thermo Scientific, Waltham, USA). DNA oligonucleotide sequences of the $-248 \sim-217$ nt probe (TTATTAAAACATCCGAAAACGCAATAATCATT) and the mutated probe (GGCGGCCCACATCCGAAA ACGACCGCCGACGG) were heated at $95^{\circ} \mathrm{C}$ for $10 \mathrm{~min}$ in $50 \mathrm{mM}$ Tris buffer at $\mathrm{pH} 7.5$ and slowly cooled to room temperature over $4 \mathrm{~h}$ to generate the doublestranded probe. Double-stranded biotinylated DNA oligonucleotide $(20 \mu \mathrm{g})$ was incubated with $100 \mu \mathrm{g}$ streptavidin-coated Dynabeads (Life Technologies, USA) in $400 \mu \mathrm{L}$ binding buffer $(10 \mathrm{mM}$ Tris, $\mathrm{pH} 7.5,1 \mathrm{mM}$ EDTA, $1 \mathrm{M} \mathrm{NaCl}, 0.003 \% \mathrm{NP} 40$ ) for $30 \mathrm{~min}$ at room temperature with constant and slow rotation. After twice washing with binding buffer, the immobilized DNA was incubated for $30 \mathrm{~min}$ in $400 \mu \mathrm{L}$ blocking buffer $(2.5 \mathrm{mg}$ / $\mathrm{ml}$ BSA, $10 \mathrm{mM}$ HEPES, $\mathrm{pH}$ 7.6, $100 \mathrm{mM}$ potassium glutamate, $2.5 \mathrm{mM}$ DTT, $10 \mathrm{mM}$ magnesium acetate, $5 \mathrm{mM}$ EGTA, 3.5\% glycerol with $0.003 \% \mathrm{NP} 40$, and $5 \mathrm{mg} / \mathrm{ml}$ polyvinylpyrrolidone). Then, $100 \mu \mathrm{g}$ of nuclear proteins were incubated with the immobilized DNA for $4 \mathrm{~h}$ at $4{ }^{\circ} \mathrm{C}$ in $400 \mu \mathrm{L}$ protein binding buffer (10 mM HEPES, $\mathrm{pH} 7.6,100 \mathrm{mM}$ potassium glutamate, $80 \mathrm{mM} \mathrm{KCl}, 2.5$ $\mathrm{mM}$ DTT, $10 \mathrm{mM}$ magnesium acetate, $5 \mathrm{mM}$ EGTA, $3.5 \%$ glycerol with $0.001 \% \mathrm{NP} 40$, and $1 \mu \mathrm{g}$ non-specific DNA) with constant and slow rotation. After $4 \mathrm{~h}$, the DNA/protein complexes were washed three times with $400 \mu \mathrm{L}$ washing buffer (10 mM HEPES, pH 7.6, $100 \mathrm{mM}$ potassium glutamate, $2.5 \mathrm{mM}$ DTT, $10 \mathrm{mM}$ magnesium acetate, $5 \mathrm{mM}$ EGTA, 3.5\% glycerol, $0.5 \mathrm{mg} / \mathrm{ml}$ BSA, $0.05 \%$ NP40). The proteins bound to the DNA were then eluted in $20 \mu \mathrm{L}$ SDS-PAGE sample buffer $(50 \mathrm{mM}$ Tris, $100 \mathrm{mM}$ DTT, 2\% SDS, 0.1\% Bromophenol blue, 10\% glycerol). The eluted proteins were subjected to $12 \%$ SDS-PAGE. The gels were stained with Coomassie Brilliant Blue R-250 for more than $4 \mathrm{~h}$ and distained with distaining solution (10\% acetic acid, 5\% ethanol, $85 \%$ water). The differentiated protein bands were excised and subjected to protein identification using LC-MS/MS in Huijun Biotechnology company (Guangzhou, China).

\section{Construction of ex vitro translation system}

$B m K R P$ open reading frame (ORF) fragment was amplified and then sub-cloned into the pF25A ICE T7 Flexi vector (Promega, Madison, USA) infusion with T7 promoter between Sgf I and Pme I restriction enzyme sites, generating a BmKRP-pF25A ICE T7 Flexi recombinant expression vector. BmKRP-pF25A ICE T7 Flexi $(4 \mu \mathrm{g})$ was used as a template for ex vitro translation in the
TNT Quick Coupled Transcription/Translation System (Promega, Madison, USA) containing $40 \mu \mathrm{L}$ of TNT T7 Quick Master Mix and nuclease-free water of a final volume of $50 \mu \mathrm{L}$. The reaction was performed at $30{ }^{\circ} \mathrm{C}$ for $4 \mathrm{~h}$ and $2 \mu \mathrm{L}$ of translation product was then used for EMSA assay.

\section{Electrophoretic mobility shift assay (EMSA) for protein- DNA binding}

EMSA was conducted using the Light Shift Chemiluminescent EMSA Kit (Thermo Scientific, Waltham, USA). The wild-type and mutant oligonucleotides of -248 $-217 \mathrm{nt}$ and $-234 \sim-203 \mathrm{nt}$ probes (Fig. 3d) were labeled with biotin at the $5^{\prime}$ end and heated at $95^{\circ} \mathrm{C}$ for $10 \mathrm{~min}$ in $50 \mathrm{mM}$ Tris-acetate buffer at $\mathrm{pH} 4.1$ and slowly cooled to room temperature. The biotinlabeled oligonucleotides were synthesized by Invitrogen (Shanghai, China). Binding reactions were performed according to the instruction of the EMSA Kit. Briefly, reactions were conducted in a $20 \mu \mathrm{L}$ mix containing $(1 \times$ binding buffer, $2.5 \%$ glycerol, $0.05 \%$ $\mathrm{NP}-40,50 \mathrm{mM} \mathrm{KCl}, 5 \mathrm{mM} \mathrm{MgCl}_{2}, 4 \mathrm{mM}$ EDTA, $1 \mu \mathrm{g}$ poly dI-dC, $2 \mu \mathrm{g}$ nuclear proteins or $2 \mu \mathrm{L}$ of translated product BmKRP and $20 \mathrm{fmol}$ of a biotinylated endlabeled probe) at room temperature for $20 \mathrm{~min}$. For the competition assay, cold probes (un-biotinylated) were added to the binding reaction. The samples were then separated in $6 \%$ polyacrylamide gels on the ice at $100 \mathrm{~V}$ for $1.5 \mathrm{~h}$. After electrophoresis, the gels were blotted onto a Nylon positively charged membrane (Amersham Biosciences, Fairfield, USA). The membranes were then developed using the Light Shift Chemiluminescent EMSA kit according to the manufacturer's protocol.

\section{Subcellular localization of BmKRP protein}

For subcellular localization study of BmKRP, the ORF DNA fragment of BmKRP was PCR amplified with a FLAG tag and a His-tag between Kpn I and BamH I with forward primer: 5'- GGTACCGATGGATTACAA GGATGACGACGATAAGGGTTCAAAGATACCAG-3' and reverse primer: 5' - GGATCCCGGTGATGATGA 'TGATGATGAATTTTTATAACCATA-3' and cloned into pEGFP-N1 plasmid vector (Clontech Laboratories Inc., Mountain View, CA, USA), generating a recombinant plasmid vector, BmKRP-EGFP. BmKRP-EGFP was transfected into BmN cells. BmKRP-EGFP protein was localized by observing the green fluorescence signal under a laser confocal microscope (Carl Zeiss AG, Oberkochen, Germany) at $24 \mathrm{~h}$ after transfection. The vector expressing EGFP alone was used as control. The nuclei were counterstained by DAPI. 


\section{Chromatin immunoprecipitation (ChIP) assay}

ChIP assay was conducted to detect the binding of BmKRP with the 20E cis-response element of the $B m K r$ h1 promoter in $\mathrm{BmN}$ cells. Cells were crosslinked with $1 \%$ formaldehyde for $10 \mathrm{~min}$ at room temperature after being transfected with BmKRP-EGFP or EGFP (control) plasmids for $48 \mathrm{~h}$. Glycine was added to terminate the fixation and the cells were washed twice with one media volume of ice-cold PBS. Cells in $1 \mathrm{~mL}$ of ice-cold PBS and $10 \mu \mathrm{L} \mathrm{Halt}{ }^{\mathrm{Tm}}$ Protease Inhibitor Cocktail (Thermo Scientific, Waltham, USA) were collected by scraping. The cells were centrifuged at $3000 \mathrm{~g}$ for $5 \mathrm{~min}$ and the cell pellet was homogenized with extraction buffer containing protease/phosphatase inhibitors. Nuclei were collected by centrifuged at $9000 \mathrm{~g}$ for $3 \mathrm{~min}$ and digested by MNase and chromatin was obtained by sonication on ice with several pulses to break nuclear membrane and incubated for $20 \mathrm{~s}$ on ice between pulses and then centrifuged at $9000 \mathrm{~g}$ for $5 \mathrm{~min}$. Immunoprecipitation was performed following the manufacturer's instruction of Pierce $^{\mathrm{Tm}}$ Magnetic ChIP Kit (Thermo Scientific, Waltham, USA). Ten micrograms of either rabbit anti-EGFP antibody or normal rabbit IgG (Thermo Scientific, Waltham, USA) were used and IP reactions were incubated overnight at $4{ }^{\circ} \mathrm{C}$ with constant mixing. DNA/protein/antibody complex was purified by incubating with ChIP Grade Protein A/G Magnetic Beads for $2 \mathrm{~h}$ at $4{ }^{\circ} \mathrm{C}$ with mixing. Immunoprecipitated genomic DNA fragments were amplified by qRT-PCR with primers (Table S3). The length of the product was $101 \mathrm{bp}$. The specificity of the primers was examined using Primer-blast (http:// www.ncbi.nlm.nih.gov/tools/primer-blast/). For qRTPCR, the SYBR Green Kit was used according to the manufacturer's instruction (TaKaRa, Dalian, China) and was performed at the following conditions: SYBR Premix Ex Taq (2x): $10 \mu \mathrm{L}$ in $20 \mu \mathrm{L}$ reaction volume, the primers concentrations: $0.4 \mu \mathrm{L}(10 \mu \mathrm{M})$, the immunoprecipitated DNA samples: $4 \mu \mathrm{L}$. The mix was incubated at $95^{\circ} \mathrm{C}$ for $10 \mathrm{~s}$, followed by 40 cycles at $95^{\circ} \mathrm{C}$ for $5 \mathrm{~s}$, $60^{\circ} \mathrm{C}$ for $30 \mathrm{~s}$ using ABI7300 fluorescence quantitative PCR system. The enrichment of the promoter sequence in the immunoprecipitated DNA samples was normalized with DNA present in the $10 \%$ input material analyzed using the comparative $\mathrm{Ct}\left(2^{-\Delta \Delta \mathrm{Ct}}\right)$ method [49]. The PCR products of the enriched promoter were sequenced for confirmation.

\section{Generation of $\mathrm{Bmkrp}^{-/-}$mutants by CRISPR/Cas9}

A 23-bp sgRNA targeting site at exon I of $B m K R P$ was identified (SI Appendix, Fig. 5A) according to the GGN19GG rule [50, 51]. The sgRNA DNA template was synthesized by PCR with Q5 ${ }^{\circ}$ High-Fidelity DNA Polymerase (NEB, Beijing, China), the oligonucleotide (BmKRP-sgF1) that encoded T7 polymerase binding site,
sgRNA targeting sequence, and overlap sequence was annealed to a common oligonucleotide that encoded the remainder of the sgRNA sequence (sgRNA-R) (Table S3). The reaction conditions were as follows: $98^{\circ} \mathrm{C}$ for 2 min, 35 cycles of $94{ }^{\circ} \mathrm{C}$ for $10 \mathrm{~s}, 55^{\circ} \mathrm{C}$ for $30 \mathrm{~s}$, and $72{ }^{\circ} \mathrm{C}$ for $2 \mathrm{~min}$, followed by a final extension period of $72^{\circ} \mathrm{C}$ for $10 \mathrm{~min}$. The sgRNA was synthesized based on the DNA template in vitro with MAXIscript $^{\circ}$ T7 Kit (Ambion, Austin, TX, USA). The Cas9 gene template was provided by the Shanghai Institute of Plant Physiology and Ecology (Shanghai, China). Cas9 mRNA was prepared using the mMESSAGE mMACHINE ${ }^{\circ}$ T7 kit (Ambion, Austin, USA).

The fertilized eggs were collected and injected within $6 \mathrm{~h}$ after oviposition. A mix of Cas9 mRNA (1000 ng/ $\mu \mathrm{L})$ and sgRNA $(1000 \mathrm{ng} / \mu \mathrm{L})$ was injected into the P50 embryos (about $12 \mathrm{~nL} / \mathrm{egg}$ ) using a micro-injector (FemtoJet ${ }^{\circ}$, Germany). The injected eggs were incubated at $25^{\circ} \mathrm{C}$ for $9-10$ days until hatching. The hatched animals were G0 individuals. Genomic DNA was extracted from the larvae-sloughs of G0 individuals for genotyping by sequencing and the multiple peaks in chromatograms of the PCR-product sequencing at the region flanking the target position were indicatives of mosaic mutations. Individuals with such mosaic mutation genotypes were used to mate with the wild-type or pair-mating to produce G1 generation offspring populations. The mosaic mutation genotypes of $\mathrm{G} 1$ generation were shown in SI Appendix, Fig. S8A. Two mutants with 4 bp and 2 bp deletion were selected for pair-mating to produce G2 generation, respectively. In G3 generation, the homozygous mutants with shift-frame mutation ( 4 bp deletion and 2 bp deletion) were identified and used for phenotypic observation.

\section{Genomic DNA extraction and mutagenesis analysis}

Genomic PCR, followed by sequencing, was carried out to identify the Bmkrp mutant alleles generated by the CRISPR/Cas9 system. Genomic DNA was extracted by TIANamp Blood DNA Kit (Tiangen Biotech, Beijing, China). The PCR conditions were as follows: $94{ }^{\circ} \mathrm{C}$ for 2 min, 35 cycles of $94{ }^{\circ} \mathrm{C}$ for $30 \mathrm{~s}, 55^{\circ} \mathrm{C}$ for $45 \mathrm{~s}$, and $72{ }^{\circ} \mathrm{C}$ for $30 \mathrm{~s}$, followed by a final extension period of $72{ }^{\circ} \mathrm{C}$ for $10 \mathrm{~min}$. The amplified fragments were cloned into a pMD19-T Simple Vector (TaKARA, Dalian, China). The primers designed to detect mutagenesis in targeted sites are shown in Table S3.

\section{Analysis of 20E CRE in Kr-h1 homologs and Kr-h1 binding site in BmVgR promoter}

Genomic databases of $T$. castaneum, A. mellifera, A. aegypti, Acyrthosiphon pisum, and Drosophila melanogaster were searched for $K r-h 1$ homologs with the tblastn program (http://blast.ncbi.nlm.nih.gov/) by using the 
$B m K r$ - $h 1$ sequence (GenBank accession no.: BAJ05087) as a query. The 20E CRE (5'-TTATTAA- $\left.3^{\prime}\right)$ and (5'TTATTAA-3') located in the BmKr-h1 promoter were searched in the $5^{\prime}$-flanking regions of $K r-h 1$ in T. castaneum, A. mellifera, A. aegypti, A. pisum, and D. melanogaster. The $B m V g R$ promoter sequence was used to identify the putative $\mathrm{Kr}-\mathrm{h} 1$ binding site (KBS) in the 5 '-flanking regions by comparing the KBS in the promoters of BmE93 [3], BmBR-C [31], and steroidogenic enzyme genes [35].

\section{RNA interference (RNAi)}

For RNAi of BmKRP in BmN cells, a 431-bp unique fragment between 170 and 600 nt in the ORF of BmKRP was used as a template for synthesizing gene-specific dsRNA from the linearized template by using the T7 RiboMAXTM Express RNAi System (Promega, Madison, USA). Total RNA extraction and reverse transcription were conducted as above mentioned. RT-PCR was performed using specific primers designed based on the $B m K R P$ cDNA to detect the knockdown efficiency. To detect the effect of $B m K R P$ on $B m K r-h 1$ promoter activity when $B m K R P$ was knocked down, a $50-\mu \mathrm{L}$ mixture containing $2.5 \mu \mathrm{g} B m K R P$ dsRNA or EGFP dsRNA in 5$\mu \mathrm{L}$ Fugene HD transfection reagent in the Opti-MEM Reduced Serum Medium (Invitrogen, CA, USA) was added to cells. After $24 \mathrm{~h}$ dsRNA transfection, $50 \mu \mathrm{L}$ mixture containing $0.5 \mu \mathrm{g}$ of the wild-type or mutated BmKr-h1-Promoter (-2877/+81 bp)-pGL3 reporter plasmid DNA, 50 ng internal control plasmid (pRL-SV40 vector), and $1.5-\mu \mathrm{L}$ Fugene $\mathrm{HD}$ transfection reagent in the Opti-MEM Reduced Serum Medium was added to cells and $1 \mu \mathrm{M} 20 \mathrm{E}$ was added to the cells at the final concentration at $6 \mathrm{~h}$ post second transfection. After culture for $24 \mathrm{~h}$ at $28^{\circ} \mathrm{C}$, the cells were collected for the promoter activity assay.

For RNAi of $B m K r-h 1$ in B. mori, a $533 \mathrm{bp}$ unique fragment between 1 and $532 \mathrm{nt}$ in the ORF of $B m K r-h 1$ was used as a template for synthesizing gene-specific dsRNA from the linearized template by using the T7 RiboMAXTM Express RNAi System (Promega, Madison, USA). Pupae at day 6 were used for dsRNA injection. The pupae were anesthetized on ice for $5 \mathrm{~min}$ prior to microinjection. Fifteen micrograms dsRNA per pupa was injected into the middle ventral segment, and three replicates of 70-100 pupae each were injected for each of the treatment groups. The epidermis, wing, testis, ovary, and egg were collected for RNA analysis. Each treatment sample included at least three individual larvae and was repeated three times.

\section{Western blotting}

Total proteins were isolated from ovaries during the pupal stage and the dsRNA treated pupae or oocytes at day 7 of wild-type and $B m \mathrm{krp}^{-/-}$pupae and day 1 of wild-type and $\mathrm{Bmkrp}^{-/-}$adult. Fifteen mircograms of proteins from the ovaries or oocytes was mixed with $5 \times$ loading buffer (250 mM Tris- $\mathrm{HCl} \mathrm{pH} 6.8,10 \%$ SDS, 0.5\% bromophenol blue, $50 \%$ glycerol, 5\% 2-ME) and separated on 12\% SDS-PAGE and transferred to nitrocellulose membranes. The membranes were washed in Trisbuffered saline-Tween 20 (TBST) for $1 \mathrm{~h}$ with buffer change every $20 \mathrm{~min}$ to remove SDS, blocked with $3 \%$ (wt/vol) BSA in TBST at $37^{\circ} \mathrm{C}$ for $2 \mathrm{~h}$. The membranes were washed in TBST for three times with each wash for $10 \mathrm{~min}$ and then incubated with anti-BmVg antibody with a gentle rocking at $30^{\circ} \mathrm{C}$ for $1 \mathrm{~h}$. After washing with TBST, the membranes were incubated with the second antibody alkaline phosphatase (AP)-conjugated goat anti-rabbit IgG with a gentle rocking at $30^{\circ} \mathrm{C}$ for $1 \mathrm{~h}$. Anti-BmVg antibodies were diluted to $1: 5000$ in 1\% (wt/ vol) BSA in TBST $(150 \mathrm{mM} \mathrm{NaCl}, 0.05 \%$ Tween 20,20 $\mathrm{mM}$ Tris- $\mathrm{HCl}, \mathrm{pH} 8.0$ ), and the second antibodies were diluted to 1:10,000 (Boster Biological Technology Co. Ltd.) in the same buffer. The mouse antibody of GAPD $\mathrm{H}$ or $\beta$-tubulin was used as an internal reference.

\section{Statistical analysis}

Statistical analysis was performed using Student's $t$ test and ANOVA analysis. Data is presented as mean \pm SD of three independent biological replicates and $p<0.05$ was considered as significant. Different letters above the columns indicate significance in the group difference at $p<0.05^{*}, p<0.01^{* * * *}$, and $p<0.001^{* * * *}$.

\section{Supplementary Information}

The online version contains supplementary material available at https://doi. org/10.1186/s12915-021-00952-2.

\footnotetext{
Additional file 1: Table S1. Statistics of phenotypes and reproduction of silkworm after RNAi. Table S2. Candidates of the 20E cis-response element binding proteins by LC-MS/MS. Table S3. Primers used in this study. Figure S1. qRT-PCR analysis of the expression and induced expres sion of $B m K r-h 1 \beta$ by $20 E$ in cultured ovaries and BmN cells. Figure S2. Development of eggs oviposited from the dsRNA-treated adults. Figure S3. Developmental and 20E-induced expression patterns of BmVg and $B m V g R$ in the ovaries and BmN cells. Figure S4. EMSA of the binding of the nuclear protein isolated from BmN cells overexpressing BmKr-h1EGFP with the KBS1 and KBS2 in the BmVgR promoter. Figure S5. Alignment of the putative $20 \mathrm{E}$ cis-response elements in the upstream of Kr-h1 in other insect species. Figure S6. Nucleotide and deduced amino acid sequences of BmKRP in B. mori. Figure S7. Developmental and 20Einduced expression patterns of BmKRP in the ovaries and BmN cells. Figure S8. Cas $9 / \mathrm{sgRNA}$ mediated gene editing of BmKRP in the silkworm. Additional file 2. The individual data values for Figs. 1, 3a-C, Fig. 4E-J, Fig. 5D, F-G, Fig. S3A-S3D and Fig. S7A-S7D.
} 


\section{Authors' contributions}

H.D. and Q.F. designed and monitored the project. Z.Z. performed experiments. H.D., Z.Z., C.T., and B.Q. interpreted the data. H.Y., J.X., and S.Z. prepared part of the experimental materials. H.D. and Z.Z. wrote the manuscript. Q. S and Q.F. finalized the manuscript. All authors read and approved the final manuscript.

\section{Funding}

This study was supported by grants from Guangdong Provincial Natural Science Foundation (No. 2017A030306003), Guangdong Provincial Special Support Program (No. 2017TQ04N744), and the Chinese National Natural Science Foundation (Nos. 31872969 and 32000338).

\section{Availability of data and materials}

All data generated or analyzed during this study are included in this published article and its supplementary information files. The data sets used and/or analyzed during the current study are also available from the corresponding author on reasonable request. Raw data can be found in Additional file 2 .

\section{Ethics approval and consent to participate}

Not applicable.

\section{Consent for publication}

Not applicable.

\section{Competing interests}

The authors declare no competing interests.

\section{Author details}

${ }^{1}$ Guangdong Key Laboratory of Insect Developmental Biology and Applied Technology, Guangzhou Key Laboratory of Insect Development Regulation and Application Research, Institute of Insect Science and Technology \& School of Life Sciences, South China Normal University, Guangzhou 510631 China. ${ }^{2}$ Division of Plant Sciences, University of Missouri, Columbia, MO 65211, USA.

Received: 16 August 2020 Accepted: 6 January 2021 Published online: 25 February 2021

\section{References}

1. Jindra M, Palli SR, Riddiford LM. The juvenile hormone signaling pathway in insect development. Annu Rev Entomol. 2013;58:181-204.

2. Belles X, Santos CG. The MEKRE93 (Methoprene tolerant-Krüppel homolog 1 -E93) pathway in the regulation of insect metamorphosis, and the homology of the pupal stage. Insect Biochem Mol Biol. 2014;52:60-8.

3. Kayukawa T, Jouraku A, Ito Y, Shinoda T. Molecular mechanism underlying juvenile hormone-mediated repression of precocious larval-adult metamorphosis. Proc Natl Acad Sci U S A. 2017;114:1057-62.

4. Raikhel AS, Brown MR, Belles X. Hormonal control of reproductive processes. In: Gilbert L, latrou K, Gill SS, editors. Comp Mol Insect Sci Vol. 3, Endocrinol. Amsterdam: Elsevier; 2005. p. 433-91.

5. Swevers L, latrou K. Ecdysteroids and ecdysteroid signaling pathways during insect oogenesis. In Ecdysone: Structures and Functions ed. G. Smagghe, Dordrecht, Neth.: Springer. 2009; pp.127-64

6. Swevers L. An update on ecdysone signaling during insect oogenesis. Curr Opin Insect Sci. 2019;31:8-13.

7. Santos CG, Humann FC, Hartfelder K. Juvenile hormone signaling in insect oogenesis. Curr Opin Insect Sci. 2019:31:43-8.

8. Arrese EL, Soulages JL. Insect fat body: energy, metabolism, and regulation. Annu Rev Entomol. 2010;55:207-25.

9. Tufail M, Takeda M. Insect vitellogenin/lipophorin receptors: molecular structures, role in oogenesis, and regulatory mechanisms. J Insect Physiol. 2009:55:87-103.

10. Minakuchi C, Zhou X, Riddiford LM. Krüppel homolog 1 (Kr-h1) mediates juvenile hormone action during metamorphosis of Drosophila melanogaster. Mech Dev. 2008;125:91-105.

11. Minakuchi C, Namiki T, Shinoda T. Krüppel homolog1, an early juvenile hormone response gene downstream of Methoprene-tolerant, mediates its anti-metamorphic action in the red flour beetle Tribolium castaneum. Dev Biol. 2009;325:341-50.
12. Konopova B, Smykal V, Jindra M. Common and distinct roles of juvenile hormone signaling genes in metamorphosis of holometabolous and hemimetabolous insects. Plos One. 2011;6:e28728.

13. Lozano J, Belles X. Conserved repressive function of Krüppel homolog 1 on insect metamorphosis in hemimetabolous and holometabolous species. Sci Rep. 2011:1:163.

14. Smykal V, Bajgar A, Provaznik J, Fexova S, Buricova M, Takaki K, Hodkova M, Jindra $M$, Dolezel $D$. Juvenile hormone signaling during reproduction and development of the linden bug, Pyrrhocoris apterus. Insect Biochem Mol Biol. 2014;45:69-76.

15. Kayukawa T, Murata M, Kobayashi I, Muramatsu D, Okada C, Uchino K, Sezutsu H, Kiuchi M, Tamura T, Hiruma K, Ishikawa Y, Shinoda T. Hormonal regulation and developmental role of Krüppel homolog 1, a repressor of metamorphosis, in the silkworm Bombyx mori. Dev Biol. 2014;388:48-56.

16. Pecasse F, Ruiz C, Richards G, Beck Y. Krüppel-homolog, a stage-specific modulator of the prepupal ecdysone response, is essential for Drosophila metamorphosis. Dev Biol. 2000;221:53-67.

17. Beckstead RB, Lam G, Thummel CS. The genomic response to 20hydroxyecdysone at the onset of Drosophila metamorphosis. Genome Biol. 2005;6:R99.

18. Xu J, Roy A, Palli SR. Creb-binding protein plays key roles in juvenile hormone action in the red flour beetle, Tribolium castaneum. Sci Rep. 2018; $8: 1426$.

19. Zhang WN, Ma L, Liu C, Chen L, Xiao HJ, Liang GM. Dissecting the role of Krüppel homolog 1 in the metamorphosis and female reproduction of the cotton bollworm, Helicoverpa armigera. Insect Mol Biol. 2018;27:492-504.

20. Wang HB, Ali SM, Moriyama M, Iwanaga M, Kawasaki H. 20hydroxyecdysone and juvenile hormone analog prevent precocious metamorphosis in recessive trimolter mutants of Bombyx mori. Insect Biochem Mol Biol. 2012:42:102-8.

21. Song JS, Wu ZX, Wang ZM, Deng S, Zhou ST. Kruppel-homolog 1 mediates juvenile hormone action to promote vitellogenesis and oocyte maturation in the migratory locust. Insect Biochem Mol Biol. 2014;52:94-101.

22. Lin XD, Yao Y, Wang B. Methoprene-tolerant (Met) and Krüpple-homologue $1(\mathrm{Kr}-\mathrm{h} 1)$ are required for ovariole development and egg maturation in the brown plant hopper. Sci Rep. 2015:5:18064.

23. Ojani R, Fu X, Ahmed T, Liu P, Zhu J. Krüpple-homologue 1 acts as a repressor and an activator in the transcriptional response to juvenile hormone in adult mosquitoes. Insect Mol Biol. 2018;27:268-78.

24. Yue Y, Yang RL, Wang WP, Zhou QH, Chen EH, Yuan GR, Wang JJ, Dou W. Involvement of Met and $\mathrm{Kr}$-h1 in $\mathrm{JH}$-mediated reproduction of female Bactrocera dorsalis (Hendel). Front Physiol. 2018:9:482

25. Hu K, Tian P, Yang L, Tang Y, Qiu L, He HL, Ding WB, Li YZ. Molecular characterization of the Krüppel-homolog 1 and its role in ovarian development in Sogatella furcifera (Hemiptera: Delphacidae). Mol Biol Rep. 2020;47:1099-106

26. Parthasarathy $\mathrm{R}$, Sun Z, Bai H, Palli SR. Juvenile hormone regulation of vitellogenin synthesis in the red four beetle, Tribolium castaneum. Insect Biochem Mol Biol. 2020:40:405-14.

27. Gujar H, Palli SR. Juvenile hormone regulation of female reproduction in the common bed bug, Cimex lectularius. Sci Rep. 2016;6:35546.

28. Kayukawa T, Minakuchi C, Namiki T, Togawa T, Yoshiyama M, Kamimura M, Mita K, Imanishi S, Kiuchi M, Ishikawa Y, Shinoda T. Transcriptional regulation of juvenile hormone-mediated induction of Krüppel homolog 1, a repressor of insect metamorphosis. Proc Natl Acad Sci U S A. 2012; 109:11729-34.

29. Gu SH, Chow YS. Analysis of ecdysteroidogenic activity of the prothoracic glands during the last larval instar of the silkworm, Bombyx mori. Insect Biochem Mol Biol. 2005;30:499-505.

30. Ohnishi E, Chatani F. Biosynthesis of ecdysone in the isolated abdomen of the silkworm, Bombyx mori. Dev Growth Differ. 1997;19:67-70.

31. Kayukawa T, Nagamine K, Ito Y, Nishita Y, Ishikawa Y, Shinoda T. Krüppel homolog 1 inhibits insect metamorphosis via direct transcriptional repression of broad-complex, a pupal specifier gene. J Biol Chem. 2016;291:1751-62.

32. Zhu ZD, Hu QH, Tong CM, Yang HG, Zheng SC, Feng QL, Deng HM. Transcriptomic analysis revealed the regulation network of BmKrüppel homolog1 in the oocyte development of Bombyx mori. Insect Sci. 2020. https://doi.org/10.1111/1744-7917.12747.

33. Antoniewski C, Laval M, Lepesant JA. Structural features critical to the activity of an ecdysone receptor binding site. Insect Biochem Mol Biol. 1993; 23:105-14. 
34. Liu S, Li K, Gao Y, Liu X, Chen W, Ge W, Feng Q, Palli SR, Li S. Antagonistic actions of juvenile hormone and 20-hydroxyecdysone within the ring gland determine developmental transitions in Drosophila. Proc Natl Acad Sci U S A. 2018:115:139-44.

35. Zhang T, Song W, Li Z, Qian W, Wei L, Yang Y, Wang W, Zhou X, Meng M, Peng J, Xia Q, Perrimon N, Cheng D. Krüppel homolog 1 represses insect ecdysone biosynthesis by directly inhibiting the transcription of steroidogenic enzymes. Proc Natl Acad Sci U S A. 2018;115:3960-5.

36. Tsuchida K, Nagata M, Suzuki A. Hormonal control of ovarian development in the silkworm, Bombyx mori. Arch Insect Biochem Biophys. 1987;5:167-77

37. Roy S, Saha TT, Zou Z, Raikhel AS. Regulatory pathways controlling female insect reproduction. Annu Rev Entomol. 2018;63:489-511.

38. Jiang J, Xu Y, Lin X. Role of Broad-Complex (Br) and Krüppel homolog 1 (Kr-h1) in the ovary development of Nilaparvata lugens. Front Physiol. 2017;8:1013.

39. Mao Y, Li Y, Gao H, Lin X. The direct interaction between E93 and Kr-h1 mediated their antagonistic effect on ovary development of the brown planthopper. Int J Mol Sci. 2019;20:2431.

40. Glinka A, Wyatt G. Juvenile hormone activation of gene transcription in locust fat body. Insect Biochem Mol Biol. 1996;26:13-1.

41. Luo M, Li D, Wang Z, Guo W, Kang L, Zhou S. Juvenile hormone differentially regulates two Grp78 genes encoding protein chaperones required for insect fat body cell homeostasis and vitellogenesis. J Biol Chem. 2017;292:8823-34

42. Comas D, Piulachs MD, Belles X. Fast induction of vitellogenin gene expression by juvenile hormone III in the cockroach Blattella germanica (L.) (Dictyoptera, Blattellidae). Insect Biochem Mol Biol. 1999:29:821-7.

43. Comas D, Piulachs MD, Belles $X$. Induction of vitellogenin gene transcription in vitro by juvenile hormone in Blattella germanica. Mol Cell Endocrinol. 2001;183:93-100

44. Marchal E, Hult EF, Huang J, Pang Z, Stay B, Tobe SS. Methoprene-tolerant (Met) knockdown in the adult female cockroach, Diploptera punctata, completely inhibits ovarian development. PLoS One. 2014;9:e106737.

45. Lu K, Chen X, Liu WT, Zhang XY, Chen MX, Zhou Q. Nutritional signaling regulates vitellogenin synthesis and egg development through juvenile hormone in Nilaparvata lugens (Stål). Int J Mol Sci. 2016;17:269.

46. Parthasarathy R, Sheng Z, Sun Z, Palli SR. Ecdysteroid regulation of ovarian growth and oocyte maturation in the red flour beetle, Tribolium castaneum. Insect Biochem Mol Biol. 2010;40:429-39.

47. Sorge D, Nauen R, Range S, Hoffmann KH. Regulation of vitellogenesis in the fall armyworm, Spodoptera frugiperda (Lepidoptera: Noctuidae). J Insect Physiol. 2000;46:969-76.

48. Shirai H, Kamimura M, Yamaguchi J, Imanishi S, Kojima T, Fujiwara H. Two adjacent cis-regulatory elements are required for ecdysone response of ecdysone receptor (ECR) B1 transcription. Plos One. 2012;7:e49348.

49. Livak K, Schmittgen TD. Analysis of relative gene expression data using real time quantitative PCR and the $2^{(-\Delta \Delta C(T))}$ method. Methods. 2001;25:402-8.

50. Fu YF, Sander JD, Reyon D, Cascio VM, Joung JK. Improving CRISPR-Cas nuclease specificity using truncated guide RNAs. Nat Biotechnol. 2014;32: 279-84

51. Wang $Y$, Li Z, Xu J, Zeng B, Ling L, You L, Chen Y, Huang Y, Tan A. The CRIS PR/Cas system mediates efficient genome engineering in Bombyx mori. Cell Res. 2013;23:1414-6.

\section{Publisher's Note}

Springer Nature remains neutral with regard to jurisdictional claims in published maps and institutional affiliations.

Ready to submit your research? Choose BMC and benefit from:

- fast, convenient online submission

- thorough peer review by experienced researchers in your field

- rapid publication on acceptance

- support for research data, including large and complex data types

- gold Open Access which fosters wider collaboration and increased citations

- maximum visibility for your research: over $100 \mathrm{M}$ website views per year

At BMC, research is always in progress.

Learn more biomedcentral.com/submissions 